\title{
Membrane flutter induced by radiation of surface gravity waves on a uniform flow
}

\author{
Joris Labarbe $^{1}$ and Oleg N. Kirillov ${ }^{1} \dagger$ \\ ${ }^{1}$ Northumbria University, Newcastle upon Tyne NE1 8ST, UK
}

(Received 23 April 2020; revised 13 June 2020; accepted 26 June 2020)

We consider the stability of an elastic membrane on the bottom of a uniform horizontal flow of an inviscid and incompressible fluid of finite depth with free surface. The membrane is simply supported at the leading and the trailing edges which attach it to the two parts of the horizontal rigid floor. The membrane has an infinite span in the direction perpendicular to the direction of the flow and a finite length in the direction of the flow. For the membrane of infinite length we derive a full dispersion relation that is valid for arbitrary depth of the fluid layer and find conditions for the flutter of the membrane due to emission of surface gravity waves. We describe this radiation-induced instability by means of the perturbation theory of the roots of the dispersion relation and the concept of negative energy waves and discuss its relation to the anomalous Doppler effect.

Key words: flow-structure interactions

\section{Introduction}

Flutter of membranes has been a classical subject for at least seven decades. Membranes submerged in a compressible gas flow occupying a space or a semi-space and their flutter at supersonic speeds have been considered already in the works by Miles (1947, 1956), Goland \& Luke (1954), Benjamin (1963) and Bolotin (1963).

Bolotin (1963), Spriggs, Messiter \& Anderson (1969), Dowell \& Ventres (1970) and Kornecki, Dowell \& O'Brien (1976) addressed the problem of the so-called membrane flutter paradox regarding the relation of stability criteria for an elastic plate to those for a membrane. Gislason (1971) demonstrated both theoretically and experimentally that a membrane or elastic plate with a finite chord develops not only flutter but also a divergence instability.

Dowell (1966), when critically appraising the study by Miles (1956) of an infinitely long, infinitely wide panel in a compressible flow occupying the upper semi-space, pointed out that the critical wavelength predicted in this study was infinite and the flutter velocity was zero, which was not physically meaningful. This observation has led him to the conclusion that the finite dimension of a membrane or a plate in the flow or a span direction is critical to the physically meaningful prediction of the instability (Dowell 1966). A similar effect

$\dagger$ Email address for correspondence: oleg.kirillov@ northumbria.ac.uk 
of an elastic foundation was shown both theoretically and experimentally by Dugundji, Dowell \& Perkin (1963).

Absolute and convective hydroelastic instabilities of slender elastic structures submerged in a uniform flow were discussed by Triantafyllou (1992). A comprehensive monograph by Dowell (2015) is a standard reference in the field.

Recent works on membrane flutter are motivated by such diverse applications as stability of membrane roofs in civil engineering (Sygulski 2007), flutter of travelling paper webs (Banichuk et al. 2010, 2019), aerodynamics of sails and membrane wings of natural flyers (Newman \& Paidoussis 1991; Tiomkin \& Raveh 2017), as well as the design of piezoaeroelastic systems for energy harvesting (Mavroyiakoumou \& Alben 2020).

Surface gravity waves on a motionless fluid of finite depth are a classical subject as well, going back to the seminal studies of Russell and Kelvin (Carusotto \& Rousseaux 2013). Numerous generalizations are known taking into account, for instance, a uniform or a shear flow and surface tension (Maissa, Rousseaux \& Stepanyants 2016), submerged solids (Smorodin 1972; Arzhannikov \& Kotelnikov 2016) and hydrofoils (Faltinsen \& Semenov 2008), a flexible bottom (Mohapatra \& Sahoo 2011) or a flexible plate resting on a free surface (Greenhill 1886; Schulkes, Hosking \& Sneyd 1987; Bochkarev, Lekomtsev \& Matveenko 2016; Das, Sahoo \& Meylan 2018a,b; Das et al. 2018). The latter setting has a straightforward motivation in the dynamics of sea ice and a less obvious application in the analogue gravity experiments (Barcelo, Liberati \& Visser 2011; Weinfurtner et al. 2011; Carusotto \& Rousseaux 2013). Recent work (Robertson \& Rousseaux 2018) discusses the effects of viscous dissipation of surface gravity waves to the analogue gravity.

Remarkably, another phenomenon that is analysed from the analogue gravity perspective is super-radiance (Barcelo et al. 2011; Carusotto \& Rousseaux 2013; Brito, Cardoso \& Pani 2015) and its particular form, discovered by Ginzburg \& Frank (1947) and Ginzburg (1996), known as the anomalous Doppler effect (ADE) (Nezlin 1976; Nemtsov \& Eidman 1987; Bekenstein \& Schiffer 1998). In electrodynamics, the ADE manifests itself when an electrically neutral overall particle, endowed with an internal structure, becomes excited and emits a photon during its uniform but superluminal motion through a medium, even if it started the motion in its ground state; the energy source is the bulk motion of the particle (Bekenstein \& Schiffer 1998).

The anomalous Doppler effect in hydrodynamics was demonstrated for a mechanical oscillator with one degree of freedom, moving parallel to the border between two incompressible fluids of different densities (Gaponov-Grekhov, Dolina \& Ostrovskii 1983). It was shown that the oscillator becomes excited due to radiation of internal gravity waves if it moves sufficiently fast. In Abramovich, Mareev \& Nemtsov (1986) the ADE for such an oscillator was demonstrated due to radiation of surface gravity waves in a layer of an incompressible fluid.

Nemtsov (1985) was the first who considered flutter of an elastic membrane resting at the bottom of a uniform horizontal flow of an inviscid and incompressible fluid as an anomalous Doppler effect due to emission of long surface gravity waves. In the shallow water approximation, he investigated both the case of a membrane that spreads infinitely far in both horizontal directions and the case when the length of the membrane in the direction of the flow (or the chord length) is finite whereas the span in the perpendicular direction is infinite. Nevertheless, the case of flow of arbitrary depth has not been studied in Nemtsov (1985), and no numerical computation supporting the asymptotic results has been performed. Another issue that has not been addressed in Nemtsov (1985) is the relation of stability domains for the membrane of finite length to those for the membrane of infinite length. 
Vedeneev (2004) studied flutter of an elastic plate of finite and infinite length at the bottom of a uniform horizontal flow of a compressible gas occupying the upper semi-space. He performed an analysis of the relation of the stability conditions for the finite plate to those for the infinite plate using the method of global stability analysis of Kulikovskii (Doaré \& de Langre 2006; Vedeneev 2016). A single-mode high-frequency flutter due to a negative aerodynamic damping and a binary flutter have been identified in Vedeneev (2016). However, no connection has been made to the ADE and the concept of negative energy waves.

In the present work we reconsider the setting of Nemtsov in order to address the finite depth of the fluid layer, find flutter domains in the parameter space, analyse them using perturbation of multiple roots of the dispersion relation and investigate the flutter onset for the membrane of infinite chord length. We will explain the radiative instabilities via the interaction of positive and negative energy waves using an explicit expression for the averaged total energy derived rigorously from physical considerations and relate them to the anomalous Doppler effect. We believe that the Nemtsov membrane is as important for understanding the phenomenon of radiation-induced instabilities (Hagerty, Bloch \& Weinstein 2003) as the famous Lamb oscillator coupled to a semi-infinite string was for understanding the radiative damping (Lamb 1900; Barbone \& Crighton 1994).

\section{Model of a membrane interacting with a free surface}

\subsection{Physical system}

In a Cartesian coordinate system $O X Y Z$, consider an inextensible elastic rectangular membrane strip of constant thickness $h$ and density $\rho_{m}$, of infinite span in the $Y$-direction, held at $Z=0$ at the leading edge $(X=0)$ and at the trailing edge $(X=L)$ by simple supports, figure 1 .

The membrane is initially still and flat, immersed in a layer of inviscid, incompressible fluid of constant density $\rho$, with free surface at the height $Z=H$. The two-dimensional flow in the layer is supposed to be irrotational and moving steadily with velocity $v$ in the positive $X$-direction. The bottom of the fluid layer at $Z=0$ is supposed to be rigid and flat for $X \in(-\infty, 0] \cup[L,+\infty)$.

Nemtsov (1985) assumed that a vacuum exists below the membrane. In the present study we prefer to consider that a motionless incompressible medium of the same density $\rho$ is present below the membrane with a pressure that is the same as the unperturbed pressure of the fluid (Vedeneev 2004, 2016).

Assuming small vertical displacement of the membrane $w(X, t)$, where $t$ is time, a constant tension $T$ along the membrane profile and neglecting viscous forces, we write the dimensional membrane dynamic equation as (Tiomkin \& Raveh 2017)

$$
\rho_{m} h \partial_{t}^{2} w=T \partial_{X}^{2} w-\Delta P, \quad X \in[0, L], Z=0,
$$

where $\Delta P(X, t)$ is the pressure difference across the interface $Z=0$. The simply supported boundary conditions for the membrane are

$$
w(0)=w(L)=0 \quad \text { at } Z=0 .
$$

In general, to recover the pressure $P(X, Z, t)$ of the fluid we write the Euler equation for the vorticity-free flow (Carusotto \& Rousseaux 2013; Maissa et al. 2016)

$$
\partial_{t} \boldsymbol{v}+\nabla\left(\frac{\boldsymbol{v} \cdot \boldsymbol{v}}{2}+\frac{P}{\rho}+g Z\right)=0
$$




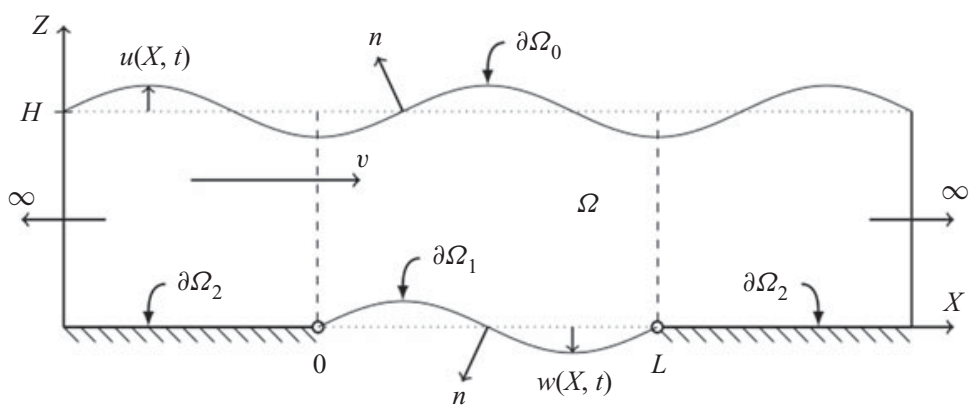

FIgURE 1. An elastic membrane with chord of length $L$ attached to two rigid walls along its leading $(X=0)$ and trailing $(X=L)$ edges on the bottom of a fluid layer of depth $H$ moving with the velocity $v . \Omega$ is the fluid domain and $\partial \Omega_{0}, \partial \Omega_{1}$ and $\partial \Omega_{2}$ are respectively the free surface, membrane and rigid wall boundaries.

with $\boldsymbol{v}=v \boldsymbol{e}_{X}+\nabla \varphi$, where $\varphi(X, Z, t)$ is the potential of the fluid, $\boldsymbol{e}_{X}$ is the unit vector in the $X$-direction and $g$ stands for the gravitational acceleration. This yields the integral of Bernoulli

$$
\frac{P}{\rho}+\left(\partial_{t}+v \partial_{X}\right) \varphi+\frac{1}{2} \nabla \varphi \cdot \nabla \varphi+g Z=\text { const. }
$$

The incompressibility condition takes the form

$$
\nabla^{2} \varphi=0
$$

From (2.4) it follows that, in the case when a motionless medium of density $\rho$ is present below the membrane with its pressure equal to the unperturbed pressure of the fluid above the membrane, the linear in $\varphi$ expression for the pressure difference, $\Delta P(X, t)$, is

$$
\Delta P(X, t)=-\rho\left(\partial_{t}+v \partial_{X}\right) \varphi(X, 0, t) .
$$

For the sake of completeness, we present also the analogous expression for the pressure difference for the case when there is a vacuum below the membrane (Nemtsov 1985)

$$
\Delta P(X, t)=-\rho\left(\partial_{t}+v \partial_{X}\right) \varphi(X, 0, t)-\rho g w(X, t) .
$$

Impermeability of the rigid bottom implies the condition

$$
\nabla \varphi \cdot \boldsymbol{n}=0 \quad \text { at } Z=0, X \in(-\infty, 0] \cup[L,+\infty) .
$$

The prescription of the normal velocity at the boundaries of moving surfaces allows us to express the kinematic condition for the membrane

$$
\nabla \varphi \cdot \boldsymbol{n}=-\left(\partial_{t}+v \partial_{X}\right) w \quad \text { at } Z=0, X \in[0, L],
$$

and to specify the same condition at the free surface

$$
\nabla \varphi \cdot \boldsymbol{n}=\left(\partial_{t}+v \partial_{X}\right) u
$$

where $u(X, t)$ is the free surface elevation and $\boldsymbol{n}$ is the vector of the outward normal to a surface. This implies that the projection of the vector $\nabla \varphi$ to the normal will coincide with the positive $z$-direction for the free surface and have the opposite direction for the membrane, see figure 1 . 
Using the Bernoulli integral (2.4) at the free surface where $P=0$ and retaining only linear in $\varphi$ terms, we find

$$
g u=-\left(\partial_{t}+v \partial_{X}\right) \varphi .
$$

Taking $u$ from (2.11) and substituting it into (2.10) we obtain the boundary condition at the free surface of the liquid that reads

$$
\nabla \varphi \cdot \boldsymbol{n}=-\frac{1}{g}\left(\partial_{t}+v \partial_{X}\right)^{2} \varphi \quad \text { at } Z=H .
$$

\subsection{Dimensionless mathematical model}

Let us choose the height of the fluid layer, $H$, as a length scale, and $\omega_{0}^{-1}$, where $\omega_{0}=$ $\sqrt{g / H}$, as a time scale. Then, we can introduce the dimensionless time and coordinates

$$
\tau=t \omega_{0}, \quad x=\frac{X}{H}, \quad y=\frac{Y}{H}, \quad z=\frac{Z}{H},
$$

the dimensionless variables

$$
\xi=\frac{w}{H}, \quad \eta=\frac{u}{H}, \quad \phi=\frac{\omega_{0}}{g H} \varphi
$$

the dimensionless parameters of the added mass ratio (Minami 1998) and membrane chord length

$$
\alpha=\frac{\rho H}{\rho_{m} h}, \quad \Gamma=\frac{L}{H},
$$

and the two Mach numbers (Vedeneev 2004, 2016)

$$
M_{w}=\frac{c}{\sqrt{g H}}, \quad M=\frac{v}{\sqrt{g H}},
$$

where $c^{2}=T /\left(\rho_{m} h\right)$ is the squared speed of propagation of elastic waves in the membrane and $\sqrt{g H}$ is the speed of propagation of long surface gravity waves in the shallow water approximation. The added mass ratio $\alpha$ is the ratio of the fluid to solid mass contained in the volume delimited by the dashed lines in figure 1 and in the membrane (Minami 1998). In figure $1, \Omega$ denotes the fluid domain and $\partial \Omega_{0}, \partial \Omega_{1}$ and $\partial \Omega_{2}$ stand, respectively, for the free surface, membrane and solid wall borders.

The dimensionless wave equation (2.1) is therefore

$$
\partial_{\tau}^{2} \xi-M_{w}^{2} \partial_{x}^{2} \xi=-\alpha \frac{\Delta P}{\rho g H}, \quad x \in[0, \Gamma], z=0 .
$$

Supplementing it with the expression (2.6), which in the dimensionless time and coordinates has the form

$$
\frac{\Delta P}{\rho}=-\left(\omega_{0} \partial_{\tau}+\frac{v}{H} \partial_{x}\right) \varphi
$$

we find

$$
\begin{aligned}
\partial_{\tau}^{2} \xi-M_{w}^{2} \partial_{x}^{2} \xi & =\alpha\left(\partial_{\tau}+\frac{v}{\omega_{0} H} \partial_{x}\right) \frac{\omega_{0}}{g H} \varphi \\
& =\alpha\left(\partial_{\tau}+M \partial_{x}\right) \phi
\end{aligned}
$$


The dimensionless boundary condition (2.9) is

$$
\nabla \phi \cdot \boldsymbol{n}=-\left(\partial_{\tau}+M \partial_{x}\right) \xi \quad \text { at } z=0, x \in[0, \Gamma],
$$

whereas the boundary condition (2.12) at the free surface in dimensionless form becomes

$$
\nabla \phi \cdot \boldsymbol{n}=-\left(\partial_{\tau}+M \partial_{x}\right)^{2} \phi \quad \text { at } z=1 .
$$

Collecting together (2.19)-(2.21) and the obvious dimensionless versions of (2.5) and (2.8) and assuming a time dependence in the form of $\phi, \xi \sim \mathrm{e}^{-\mathrm{i} \omega \tau}$ results in the following dimensionless set of equations and the boundary conditions for the case when a motionless medium is present below the membrane:

$$
\begin{gathered}
\nabla^{2} \phi=0, \quad \text { in } \Omega, \\
\nabla \phi \cdot \boldsymbol{n}=-\left(-\mathrm{i} \omega+M \partial_{x}\right)^{2} \phi, \quad \text { on } \partial \Omega_{0}, \\
\nabla \phi \cdot \boldsymbol{n}=V(x), \quad \text { on } \partial \Omega_{1}, \\
\boldsymbol{\nabla} \phi \cdot \boldsymbol{n}=0, \quad \text { on } \partial \Omega_{2}, \\
{\left[\omega^{2}+M_{w}^{2} \partial_{x}^{2}\right] \xi=-\alpha\left(-i \omega+M \partial_{x}\right) \phi, \quad \text { on } \partial \Omega_{1},} \\
\xi(0)=\xi(\Gamma)=0, \quad \text { on } \partial \Omega_{1},
\end{gathered}
$$

where $V(x)=\left(\mathrm{i} \omega-M \partial_{x}\right) \xi(x), x \in[0, \Gamma]$ is the impermeability condition for the membrane. For simplicity, we retain the same notation for the membrane displacement and the fluid potential after the separation of time.

Therefore, due to the irrotational, incompressible and inviscid character of the fluid, our mathematical model (2.22) consists of the Laplace equation for the fluid potential (2.22a), supplemented by the kinematic conditions for the free surface $(2.22 b)$ and the membrane $(2.22 c)$. The pressure at the surface of the fluid is also prescribed as a dynamic condition and therefore closes the system of equations for the fluid in this model: the motion of the membrane is described by a non-homogeneous wave equation $(2.22 e)$ with the pressure of the fluid (recovered through the Bernoulli principle) as a source term. The membrane is supposed to be simply supported at its extremities as in (2.22f).

\section{Methods and results}

\subsection{Membrane of infinite chord length}

Our ultimate goal is to understand the fundamentals of the phenomenon of radiation-induced instabilities in the model (2.22) that we see as a reasonable analytically treatable substitute for the famous Lamb system (Lamb 1900; Barbone \& Crighton 1994; Hagerty et al. 2003). In this paper, as a first natural step, we analyse the case when the chord of the membrane is infinite, i.e. when the membrane extends from $-\infty$ to $+\infty$ in the $x$-direction.

The extension of the Nemtsov model to the case where the fluid layer presents a finite depth is our main concern. In the following we will show that even in the limit of infinite chord length the model (2.22) demonstrates physically meaningful radiation-induced flutter that sets in at finite values of the dimensionless flow velocity $M>M_{w}>0$, no matter what the values of the wavenumber $\kappa$ and the added mass ratio $\alpha$ are, in contrast to other known models discussed, e.g. in Miles (1956) and Dowell (1966). 


\subsubsection{Dispersion relation for the fluid layer of arbitrary depth}

Since the motion of the fluid is two-dimensional in the $(x, z)$-plane and the horizontal extension of the fluid layer is infinite in the $x$-direction too, we can represent the potential of the fluid $\phi$ in the physical space by means of the inverse Fourier transform of the potential $\hat{\phi}$ in the wavenumber space as

$$
\phi(x, z, \omega)=\frac{1}{2 \pi} \int_{-\infty}^{+\infty} \hat{\phi}(\kappa, z, \omega) \mathrm{e}^{\mathrm{i} \kappa x} \mathrm{~d} \kappa,
$$

where $\kappa$ is the wavenumber and

$$
\hat{\phi}(\kappa, z, \omega)=\int_{-\infty}^{+\infty} \phi(x, z, \omega) \mathrm{e}^{-\mathrm{i} \kappa x} \mathrm{~d} x,
$$

under the standard assumption that both $\phi(x)$ and $\hat{\phi}(\kappa)$ are absolutely integrable functions, implying they vanish at infinity.

Assuming that $\partial_{x} \phi$ is also absolutely integrable, which allows us to use twice the property $\widehat{\partial_{x} \phi}=\mathrm{i} \kappa \hat{\phi}$, we find the Fourier transform of the Laplace equation (2.22a)

$$
\partial_{z}^{2} \hat{\phi}-\kappa^{2} \hat{\phi}=0
$$

The general solution to $(3.3)$ is

$$
\hat{\phi}(\kappa, z, \omega)=A(\kappa, \omega) \mathrm{e}^{\kappa z}+B(\kappa, \omega) \mathrm{e}^{-\kappa z},
$$

where $A(\kappa, \omega)$ and $B(\kappa, \omega)$ are yet to be determined from the Fourier-transformed boundary conditions.

The boundary condition $(2.22 c)$, expressing the impermeability of the membrane at $z=0$, takes the form

$$
-\partial_{z} \phi=V
$$

because the outward direction of the normal vector $\boldsymbol{n}$ to the surface of the membrane is opposite to the positive $z$-direction, see figure 1. The Fourier transform of (3.5) reads

$$
\partial_{z} \hat{\phi}=-\hat{V}(\kappa, \omega)
$$

where

$$
\begin{aligned}
\hat{V}(\kappa, \omega) & =\int_{-\infty}^{+\infty}\left(\mathrm{i} \omega \xi(s)-M \partial_{s} \xi(s)\right) \mathrm{e}^{-\mathrm{i} \kappa s} \mathrm{~d} s \\
& =\mathrm{i}(\omega-\kappa M) \hat{\xi} .
\end{aligned}
$$

Substituting (3.4) into (3.6) yields at $z=0$

$$
\kappa(A-B)=-\mathrm{i}(\omega-\kappa M) \hat{\xi} .
$$

Similarly transforming the boundary condition $(2.22 b)$ at the free surface we find

$$
\partial_{z} \hat{\phi}=(\omega-\kappa M)^{2} \hat{\phi} .
$$

Substituting (3.4) into (3.9) yields at $z=1$

$$
\kappa\left(A \mathrm{e}^{\kappa}-B \mathrm{e}^{-\kappa}\right)=(\omega-\kappa M)^{2}\left(A \mathrm{e}^{\kappa}+B \mathrm{e}^{-\kappa}\right) .
$$


Solving (3.8) and (3.10) simultaneously with respect to $A$ and $B$, we obtain

$$
\left.\begin{array}{rl}
A(\kappa, \omega) & =\frac{-\mathrm{i} \hat{\xi}\left[(\omega-\kappa M)^{2}+\kappa\right](\omega-\kappa M)}{\kappa\left[(\omega-\kappa M)^{2}-\kappa\right] \mathrm{e}^{2 \kappa}+\kappa\left[(\omega-\kappa M)^{2}+\kappa\right]}, \\
B(\kappa, \omega) & =\frac{\mathrm{i} \hat{\xi}\left[(\omega-\kappa M)^{2}-\kappa\right](\omega-\kappa M)}{\kappa\left[(\omega-\kappa M)^{2}-\kappa\right]+\kappa\left[(\omega-\kappa M)^{2}+\kappa\right] \mathrm{e}^{-2 \kappa}} .
\end{array}\right\}
$$

The Fourier transform of the non-homogeneous wave equation (2.22e) for the membrane displacement evaluated at $z=0$ reads

$$
\left(\omega^{2}-\kappa^{2} M_{w}^{2}\right) \hat{\xi}-\mathrm{i} \alpha(\omega-\kappa M) \hat{\phi}(\kappa, 0, \omega)=0 .
$$

Inserting expression (3.4) for $\hat{\phi}$ with the coefficients (3.11) into (3.12), discarding $\hat{\xi}$ in the result and introducing new parameters, namely the phase velocity

$$
\sigma=\frac{\omega}{\kappa},
$$

and the coupling parameter

$$
\beta=\frac{\alpha}{\kappa^{2}},
$$

we obtain the following dispersion equation in the case where a medium with constant pressure is present below the membrane

$$
\beta=\frac{\left(M_{w}^{2}-\sigma^{2}\right)\left[\kappa(\sigma-M)^{2}-\tanh \kappa\right]}{\kappa(\sigma-M)^{2}\left[\kappa(\sigma-M)^{2} \tanh \kappa-1\right]} .
$$

It is instructive to show another way of deriving the dispersion equation (3.15). For this, we notice that (3.6) and (3.7) allow us to express $\hat{\xi}$ by means of $\partial_{z} \hat{\phi}$. Using the result in (3.12), we can obtain a boundary condition for $\hat{\phi}(z)$ at $z=0$. This new boundary condition together with boundary condition (3.9) and equation (3.3) produce a closed-form boundary value problem for the Laplace equation with the Robin boundary conditions

$$
\left.\begin{array}{c}
\partial_{z}^{2} \hat{\phi}-\kappa^{2} \hat{\phi}=0, \\
\partial_{z} \hat{\phi}\left(\omega^{2}-\kappa^{2} M_{w}^{2}\right)-\alpha(\omega-\kappa M)^{2} \hat{\phi}=0, \quad z=0, \\
\partial_{z} \hat{\phi}-(\omega-\kappa M)^{2} \hat{\phi}=0, \quad z=1 .
\end{array}\right\}
$$

Substituting the general solution (3.4) into the boundary conditions of the problem (3.16) results in the system of two linear equations with respect to $A$ and $B$,

$$
\left.\begin{array}{c}
\kappa(A-B)\left(\omega^{2}-\kappa^{2} M_{w}^{2}\right)-\alpha(\omega-\kappa M)^{2}(A+B)=0, \\
\kappa\left(A \mathrm{e}^{\kappa}-B \mathrm{e}^{-\kappa}\right)-(\omega-\kappa M)^{2}\left(A \mathrm{e}^{\kappa}+B \mathrm{e}^{-\kappa}\right)=0 .
\end{array}\right\}
$$

This system can be written in matrix form as

$$
\left(\omega^{2} M_{1}+\omega M_{2}+M_{3}\right) f=0, \quad f:=\left(\begin{array}{c}
A \\
B
\end{array}\right),
$$


where the $2 \times 2$ matrices involved are

$$
\left.\begin{array}{c}
M_{1}=-\left(\begin{array}{cc}
\alpha-\kappa & \alpha+\kappa \\
\mathrm{e}^{\kappa} & \mathrm{e}^{-\kappa}
\end{array}\right), \\
M_{2}=2 \kappa M\left(\begin{array}{cc}
\alpha & \alpha \\
\mathrm{e}^{\kappa} & \mathrm{e}^{-\kappa}
\end{array}\right), \\
M_{3}=-\left(\begin{array}{cc}
\kappa^{2}\left(M^{2} \alpha+M_{w}^{2} \kappa\right) & \kappa^{2}\left(M^{2} \alpha-M_{w}^{2} \kappa\right) \\
\kappa \mathrm{e}^{\kappa}\left(M^{2} \kappa-1\right) & \kappa \mathrm{e}^{-\kappa}\left(M^{2} \kappa+1\right)
\end{array}\right) \cdot
\end{array}\right\}
$$

Computing the determinant of the matrix polynomial we arrive at the dispersion equation

$$
\begin{aligned}
D(\omega, \kappa) & =\operatorname{det}\left(\omega^{2} M_{1}+\omega M_{2}+M_{3}\right) \\
& =-\alpha(M \kappa-\omega)^{2}\left[(M \kappa-\omega)^{2} \tanh \kappa-\kappa\right]+\kappa\left(M_{w}^{2} \kappa^{2}-\omega^{2}\right)\left[(M \kappa-\omega)^{2}-\kappa \tanh \kappa\right] \\
& =0,
\end{aligned}
$$

which, with the notation $\sigma=\omega / \kappa$ and $\beta=\alpha / \kappa^{2}$, transforms exactly to (3.15).

For the sake of completeness we present also the dispersion relation for the system with a vacuum below the membrane

$$
\beta=\frac{\left(M_{w}^{2}-\sigma^{2}\right)\left[\kappa(\sigma-M)^{2}-\tanh \kappa\right]}{\left[\kappa^{2}(\sigma-M)^{4}-1\right] \tanh \kappa} .
$$

In the shallow water approximation corresponding to the limit $\kappa \rightarrow 0$, the expression (3.21) reduces to

$$
\beta=\left(\sigma^{2}-M_{w}^{2}\right)\left((\sigma-M)^{2}-1\right),
$$

which is nothing else but the shallow water dispersion relation derived by Nemtsov (1985).

In order to get the dispersion relation (3.21), one must take the pressure difference (2.7), make it non-dimensional and use in the expression (2.17) which then reads as

$$
\partial_{\tau}^{2} \xi-M_{w}^{2} \partial_{x}^{2} \xi-\alpha \xi=\alpha\left(\partial_{\tau}+M \partial_{x}\right) \phi
$$

After separation of time it reduces to the analogue of boundary condition $(2.22 e)$,

$$
\left[-\omega^{2}-M_{w}^{2} \partial_{x}^{2}-\alpha\right] \xi-\alpha\left(-\mathrm{i} \omega+M \partial_{x}\right) \phi(x, 0, t)=0,
$$

which has the following Fourier transform

$$
\left[\omega^{2}-\kappa^{2} M_{w}^{2}+\alpha\right] \hat{\xi}-\mathrm{i} \alpha(\omega-\kappa M) \hat{\phi}(\kappa, 0, \omega)=0 .
$$

Inserting the expression (3.4) for $\hat{\phi}$ with the coefficients (3.11) into (3.25) results, after familiar algebraic manipulations, in the dispersion relation (3.21).

\subsubsection{Analysis of the dispersion equation}

In the absence of coupling between the free surface and the membrane, i.e. for $\beta=0$, both the dispersion relation (3.15) and the dispersion relation (3.21) reduce to

$$
\left(\sigma^{2}-M_{w}^{2}\right)\left[\kappa(\sigma-M)^{2}-\tanh \kappa\right]=0,
$$

which yields the dispersion relation of the elastic waves in the free membrane $\sigma^{2}=M_{w}^{2}$ and that of the surface gravity waves on a uniform flow: $\kappa(\sigma-M)^{2}=\tanh \kappa$. The latter 
(a)

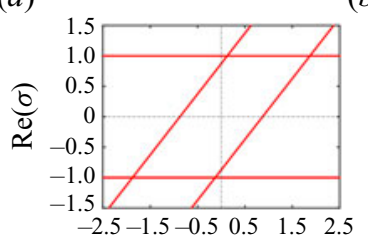

(b)

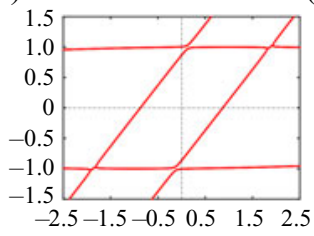

(c)

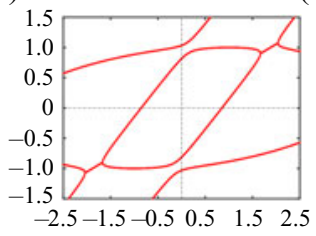

(d)

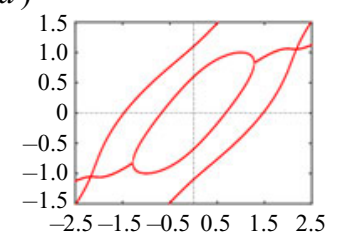

(e)

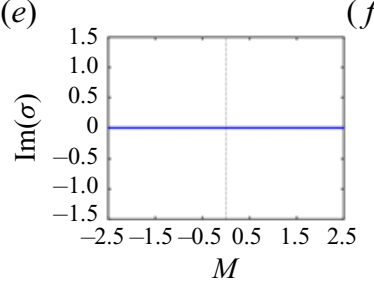

$(f)$

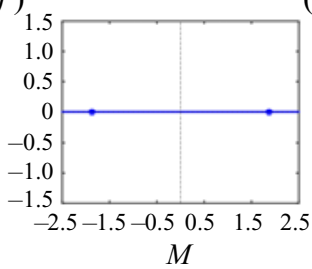

$(g)$

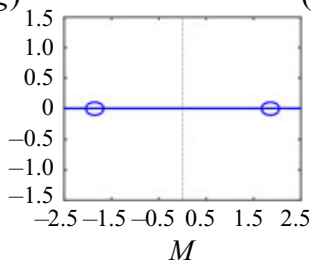

(h)

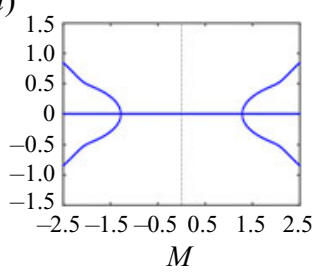

FigURE 2. Real (red, $a-d$ ) and imaginary (blue, $e-h$ ) parts of the roots of the dispersion relation (3.15) over the Mach number $M$ for $M_{w}=1, \kappa=1$ and $(a, e) \beta=0,(b, f) \beta=0.01$, $(c, g) \beta=0.1$ and $(d, h) \beta=1$.

acquires a more familiar traditional form (Maissa et al. 2016)

$$
(\omega-\kappa F r)^{2}=\kappa \tanh \kappa
$$

after taking into account that $\sigma=\omega / \kappa$ and that $M$, as defined in $(2.16 a, b)$, can also be interpreted as the Froude number, Fr.

The roots of the decoupled dispersion equation (3.26) are real

$$
\sigma_{1}^{ \pm}= \pm M_{w}, \quad \sigma_{2}^{ \pm}=M \pm \sqrt{\frac{\tanh \kappa}{\kappa}}
$$

If we consider the roots $(3.28 a, b)$ as functions of the fluid Mach number, $M$, we find that $\sigma_{1}^{ \pm}$are two horizontal straight lines and $\sigma_{2}^{ \pm}$are two straight lines with the slope equal to 1 , see figure $2(a)$. One can see that at $\beta=0$ the root branches intersect at four points forming the double roots $\sigma_{0}=M_{w}$ at

$$
M_{0}^{ \pm}=M_{w} \pm \sqrt{\frac{\tanh \kappa}{\kappa}}
$$

and the double roots $-\sigma_{0}$ at $-M_{0}^{ \pm}$. The relation $M_{w}=M_{0}^{+}-\sqrt{(\tanh \kappa) / \kappa}=\sigma_{2}^{-}=\sigma_{1}^{+}=$ $\sigma_{0}$ following from (3.29) and (3.28a,b) is the condition of 'phase synchronism' for the case of an arbitrary height of the fluid layer that extends the corresponding result obtained in Nemtsov (1985) in the shallow water limit, $\kappa \rightarrow 0$.

With the increase in $\beta$ the roots $\pm \sigma_{0}$ situated at $M= \pm M_{0}^{-}$split into simple real ones and this split is accompanied by unfolding of the crossings into avoided crossings, figure 2 .

Quite in contrast, the roots $\pm \sigma_{0}$ situated at $M= \pm M_{0}^{+}$split into complex-conjugate pairs that form bubbles of instability at moderate values of $\beta$ that open up with the increase in $\beta$ to develop disconnected complex branches, as is seen in figure $2(d, h)$. 
Let us re-write the dispersion relation (3.15) as follows

$$
\begin{aligned}
D(\sigma, M, \beta):= & \beta \kappa(\sigma-M)^{2}\left[\kappa(\sigma-M)^{2} \tanh \kappa-1\right] \\
& -\left(M_{w}^{2}-\sigma^{2}\right)\left[\kappa(\sigma-M)^{2}-\tanh \kappa\right]=0 .
\end{aligned}
$$

Then, we can apply to it the perturbation theory derived in appendix A.

Consider the double root $\sigma_{0}$ at $M=M_{0}^{+}$and $\beta=\beta_{0}=0$. Adapt the approximate equation (A 18) to our model

$$
\begin{aligned}
& \Delta \sigma\left(\partial_{\sigma M}^{2} D \Delta M+\partial_{\sigma \beta}^{2} D \Delta \beta\right)+\frac{1}{2}\left[\partial_{M}^{2} D(\Delta M)^{2}+2 \partial_{M \beta}^{2} D \Delta M \Delta \beta+\partial_{\beta}^{2} D(\Delta \beta)^{2}\right] \\
& \quad+\frac{1}{2} \partial_{\sigma}^{2} D(\Delta \sigma)^{2}+\partial_{M} D \Delta M+\partial_{\beta} D \Delta \beta=0,
\end{aligned}
$$

where $\Delta \sigma=\sigma-\sigma_{0}, \Delta M=M-M_{0}^{+}$and $\Delta \beta=\beta$. Calculating the partial derivatives at $\sigma=\sigma_{0}, M=M_{0}^{+}$, and $\beta=\beta_{0}=0$, we find

$$
\begin{gathered}
\partial_{\sigma}^{2} D=-8 M_{w} \kappa \sqrt{\frac{\tanh \kappa}{\kappa}}, \quad \partial_{\sigma M}^{2} D=4 M_{w} \kappa \sqrt{\frac{\tanh \kappa}{\kappa}}, \\
\partial_{M}^{2} D=0, \quad \partial_{M \beta}^{2} D=-\partial_{\sigma \beta}^{2} D=2 \kappa\left(2(\tanh \kappa)^{2}-1\right) \sqrt{\frac{\tanh \kappa}{\kappa}}, \quad \partial_{\beta}^{2} D=0, \\
\partial_{M} D=0, \quad \partial_{\beta} D=(\tanh \kappa)^{3}-\tanh \kappa .
\end{gathered}
$$

With the derivatives (3.32) the approximation (3.31) to the dispersion equation (3.30) near the crossing takes the form

$$
\left(\sigma-M_{w}\right)\left[\sigma-M+\sqrt{\frac{\tanh \kappa}{\kappa}}\right]=\beta \sqrt{\frac{\tanh \kappa}{\kappa}} \frac{(\tanh \kappa)^{2}-1}{4 M_{w}} .
$$

For any $\beta>0$ the crossing of the real roots $\sigma$ at $M=M_{0}^{+}$unfolds into two hyperbolic branches of the real roots

$$
\begin{gathered}
\beta \sqrt{\frac{\tanh \kappa}{\kappa}} \frac{\left[1-(\tanh \kappa)^{2}\right]}{4 M_{w}}=\frac{1}{4}\left(M-M_{w}-\sqrt{\frac{\tanh \kappa}{\kappa}}\right)^{2} \\
-\left(\operatorname{Re} \sigma-\frac{M_{w}+M}{2}+\frac{1}{2} \sqrt{\frac{\tanh \kappa}{\kappa}}\right)^{2}, \quad \operatorname{Im} \sigma=0
\end{gathered}
$$

that are connected to the 'bubble' of complex eigenvalues with the real parts $\operatorname{Re} \sigma=$ $\frac{1}{2}\left(M+M_{w}-\sqrt{(\tanh \kappa) / \kappa}\right)$ and with the imaginary parts that form an ellipse in the $(M, \operatorname{Im} \sigma)$-plane

$$
(\operatorname{Im} \sigma)^{2}+\frac{1}{4}\left(M-M_{w}-\sqrt{\frac{\tanh \kappa}{\kappa}}\right)^{2}=\beta \sqrt{\frac{\tanh \kappa}{\kappa}} \frac{\left[1-(\tanh \kappa)^{2}\right]}{4 M_{w}},
$$

see figure 3. Equating to zero the discriminant of the quadratic in $\sigma$ in equation (3.33), we arrive at the following quadratic approximation to the neutral stability curve at the crossing 


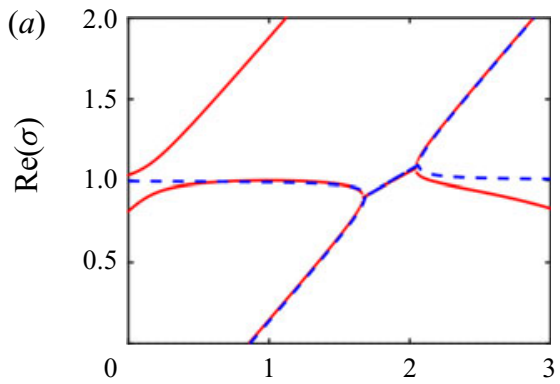

(b)
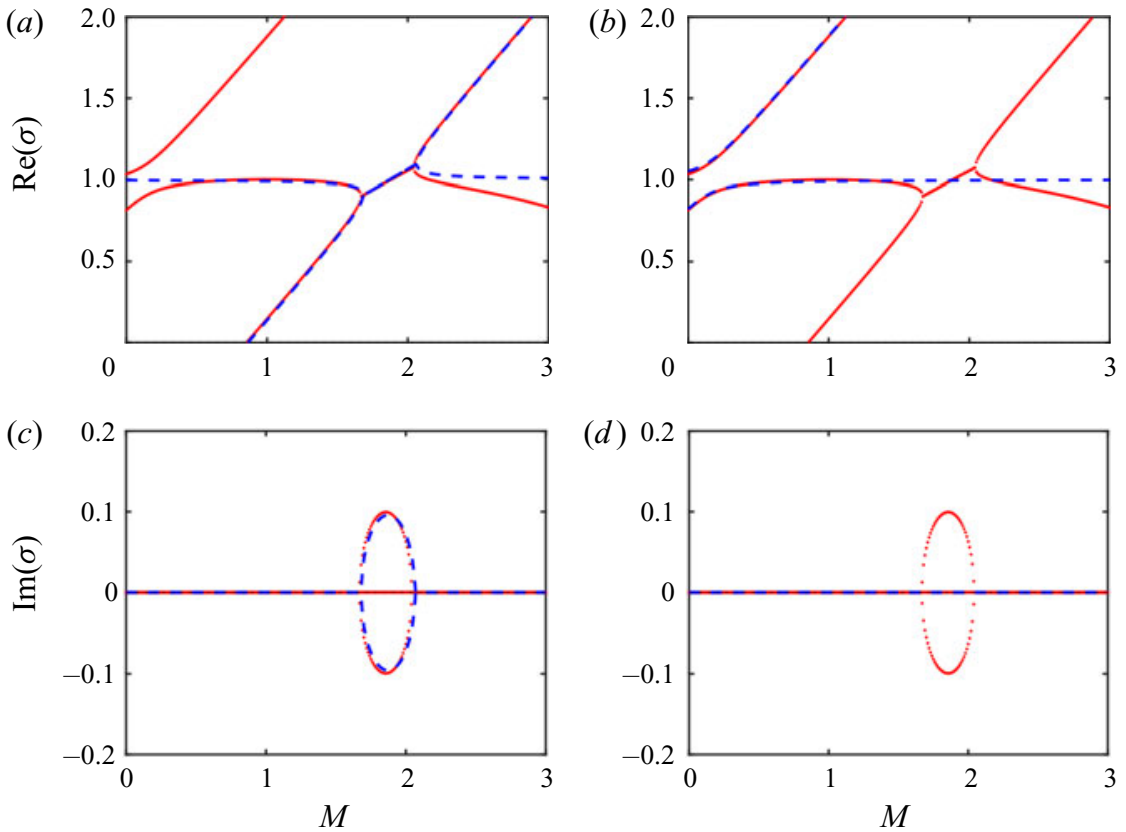

FIGURE 3. Real and imaginary parts of the roots of the dispersion relation for $M_{w}=1, \kappa=1$ and $\beta=0.1$ : (red) (3.15) and (blue, dashed) their approximations by (3.33) and (3.37) near the crossing points that exist at $\beta=0, M=M_{0}^{ \pm}, \sigma=\sigma_{0}$. Notice an avoided crossing above the line $\operatorname{Re}(\sigma)=M$ and the bubble of instability below this line.

point $M=M_{0}^{+}$

$$
\beta=M_{w} \frac{\left(M-M_{w}-\sqrt{(\tanh \kappa) / \kappa}\right)^{2}}{\left(1-(\tanh \kappa)^{2}\right) \sqrt{(\tanh \kappa) / \kappa}} .
$$

The bubble of instability (3.35) corresponds to the inner points of the instability domain bounded by (3.36).

Using the same methodology to approximate the avoided crossing close to $M=M_{0}^{-}$, $\sigma=\sigma_{0}$ and $\beta=\beta_{0}$ by (3.31), we obtain

$$
\left(\sigma-M_{w}\right)\left[\sigma-M-\sqrt{\frac{\tanh \kappa}{\kappa}}\right]=-\beta \sqrt{\frac{\tanh \kappa}{\kappa}} \frac{(\tanh \kappa)^{2}-1}{4 M_{w}} .
$$

Separating real and imaginary parts of $\sigma$ in (3.37) similarly to how it has been done in the previous case, one can see that the bubble of instability does not originate for $\beta>0$ in the unfolding of the crossing at $M=M_{0}^{-}$, see figure 3 .

In figure 4 we show that the exact neutral stability boundaries obtained from equating the discriminant of the fourth-order polynomial (3.30) in $\sigma$ to zero and their approximation (3.36) calculated at the crossing point at $M=M_{0}^{+}$are in a very good agreement.

It is instructive to change the point of view and to look at the critical values of parameters as functions of the Mach number $M_{w}$ of elastic waves in the membrane. In figure 5 we present stability maps of the dispersion equation (3.30) given by its discriminant in the $\left(M_{w}, \beta\right)$-plane for the fixed value of $M=M_{0}=2$ and increasing values of $\kappa$. We see that for all $\kappa$ the instability is possible only in the interval $\left|M_{w}\right|<M_{0}=2$, which agrees 

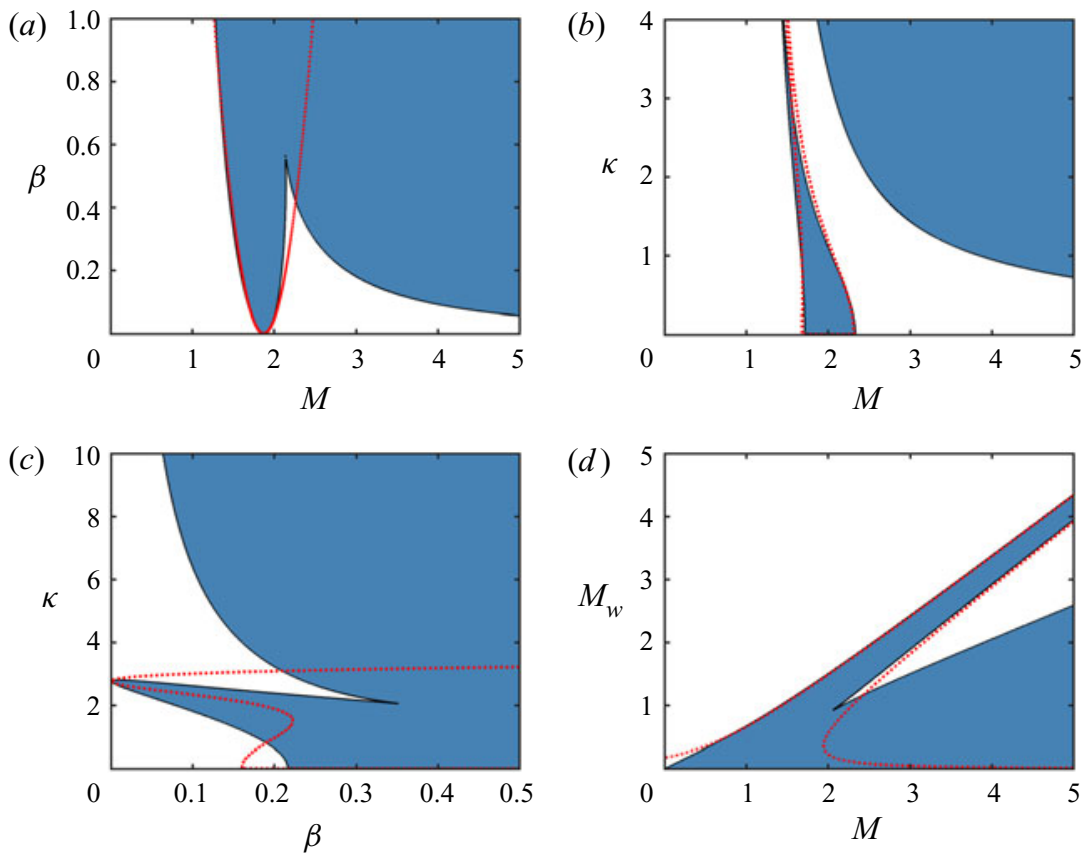

FIGURE 4. Stability maps of the dispersion equation (3.30) given by its discriminant for (a) $M_{w}=1$ and $\kappa=1,(b) M_{w}=1$ and $\beta=0.1,(c) M_{w}=1$ and $M=1.6$ and $(d) \beta=0.5$ and $\kappa=1$. The regions of real phase speed $\sigma$ are shown in white (stability) and those of the complex $\sigma$ (temporal instability) in blue. The red dotted curve is the approximation (3.36). Notice the absence of instabilities for $M_{w}>M$ in panel $(d)$.

with figure 4. For $\beta=0$, the instability domain touches the $M_{w}$-axis at the points $M_{w}=$ $M_{0}-\sqrt{(\tanh \kappa) / \kappa}$ and $M_{w}=-M_{0}+\sqrt{(\tanh \kappa) / \kappa}$. In the limit $\kappa \rightarrow 0$, the touching occurs at $M_{w}=M_{0}-1=1$ and $M_{w}=-M_{0}+1=-1$.

A qualitative change happens when $\kappa \geq \kappa_{0}$ where $\kappa_{0}>0$ is uniquely determined by $M_{0}>0$ from the equation

$$
\kappa_{0} \tanh \kappa_{0}=\frac{1}{M_{0}^{2}} .
$$

For instance, $M_{0}=2$ yields $\kappa_{0} \approx 0.5218134478$. At $\kappa=\kappa_{0}$ a new, isolated, domain of instability originates that touches the $M_{w}$-axis at $\beta=0$ and grows when $\kappa$ is further increased, figure 5. At some value of $\kappa$ the two domains touch each other and then form a unified domain. At $\kappa \rightarrow \infty$ the central part of the unified domain dominates over its side parts corresponding to the instability found by Nemtsov in the shallow water approximation when $\kappa \rightarrow 0$ and the coupling $\beta$ is weak, figure 5 .

To understand the origin of the new instability, we plot the real and imaginary values of $\sigma$ as functions of $M_{w}$ in figure 6 for a given $M=M_{0}=2$. The central panel of figure 6 corresponding to $\beta=0$ and $\kappa=\kappa_{0}$ shows four straight lines intersecting at five points, including the origin. The upper horizontal line corresponds to the fast surface gravity wave with $\sigma=M_{0}+\sqrt{\left(\tanh \kappa_{0}\right) / \kappa_{0}} \approx 3$, whereas the lower horizontal line to the slow surface gravity wave (Nemtsov 1985) with $\sigma=M_{0}-\sqrt{\left(\tanh \kappa_{0}\right) / \kappa_{0}} \approx 1$. The two inclined lines correspond to the forward and backward elastic waves in the membrane with $\sigma= \pm M_{w}$. When $\beta$ and $\kappa$ depart respectively from zero and $\kappa_{0}$, all the five crossings unfold either into 

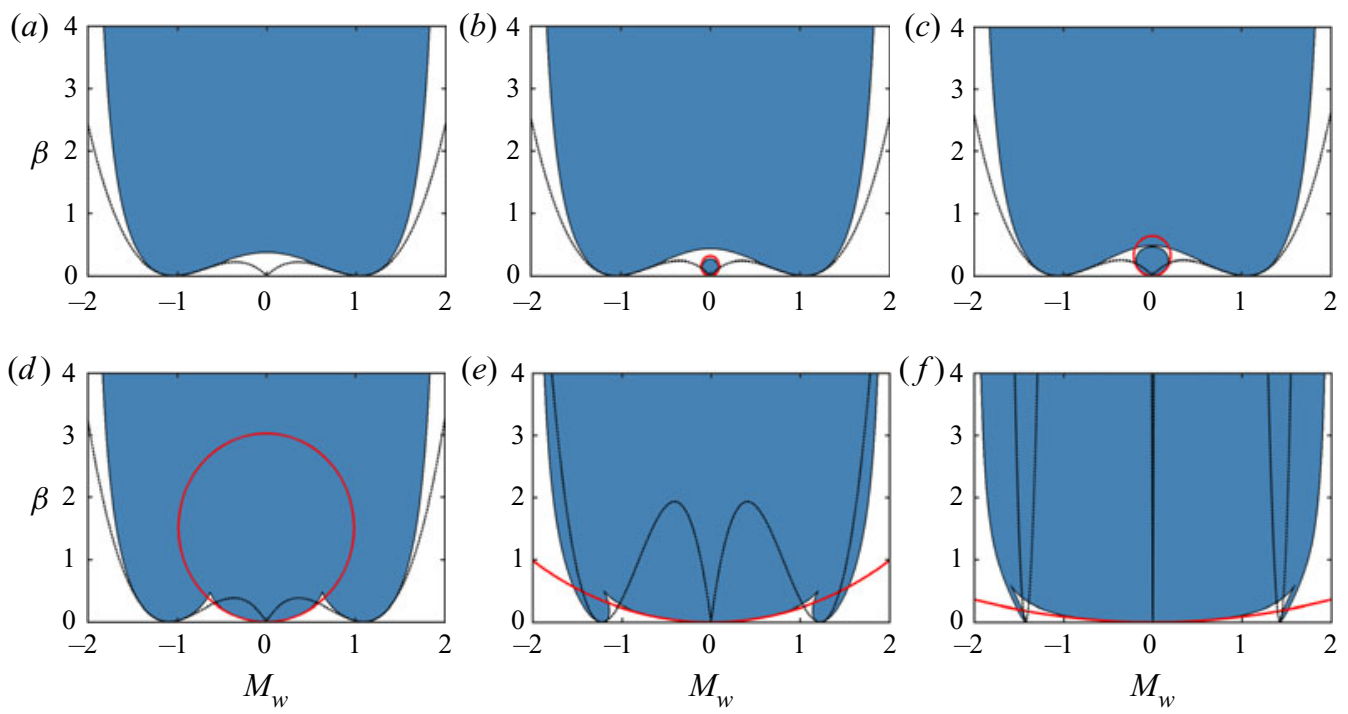

FIGURE 5. Stability maps of the dispersion equation (3.30) given by its discriminant for $M=M_{0}=2$ and: $(a) \kappa=0.5,(b) \kappa=0.55,(c) \kappa=0.58,(d) \kappa=0.8,(e) \kappa=1.5,(f) \kappa=3$. The regions of real phase speed $\sigma$ are shown in white (stability) and those of the complex $\sigma$ (temporal instability) in blue. The black dotted curve is the approximation (3.36) and the solid red ellipse is the conical approximation (3.43). When $\kappa \rightarrow \infty$, the central part of the instability domain approximated by (3.43) dominates over the side parts of the domain. Notice the absence of instabilities for $M_{w}>M_{0}$.

avoided crossings (as elastic and fast surface gravity waves) or into bubbles of instability (as elastic and slow surface gravity waves) resulting in the high-frequency flutter due to radiation of long surface gravity waves. For $\beta>0$ the crossing at the origin transforms into an avoided crossing at $\kappa<\kappa_{0}$ or into the bubble of instability at $\kappa>\kappa_{0}$, which yields low-frequency flutter at short wavelengths $\kappa$.

Figure 7(a) allows us to track the evolution of the flutter domains as $\kappa$ varies from zero to infinity at $M_{0}=2$ and $\beta=0.03$. Nemtsov's radiation-induced flutter domain is the widest in the shallow water limit and evolves along the curves (shown as black solid lines in figure $7 b$ )

$$
\left(M_{0} \pm M_{w}\right)^{2}=\frac{\tanh \kappa}{\kappa},
$$

to which the Nemtsov domains degenerate at $\beta=0$. Note that the Nemtsov flutter domain is perfectly approximated by formula (3.36) obtained from the unfolding of the eigenvalue crossing corresponding to the slow surface gravity wave and the elastic wave (dotted lines in figure $7 a$ ).

To understand the central instability domain shown in figure 7(a) for a given $\beta$ we plot it in the $\left(M_{w}, \kappa, \beta\right)$-space in figure $7(b)$, given $M=M_{0}$. One can see that the domain is symmetric with respect to the plane $M_{w}=0$ and has a pronounced conical singularity at $\kappa=\kappa_{0}$ determined by (3.38) when $\beta=0$ and $M_{w}=0$. Equation (3.38) follows from the discriminant of the dispersion equation (3.30) at $\beta=0$ and $M_{w}=0$. The conical singularity of the stability boundary therefore exactly corresponds to the crossing of the eigenvalue curves at the origin in figure $6(b)$. Usually, the conical singularity of the stability boundary is associated with a double semi-simple eigenvalue with two linearly 

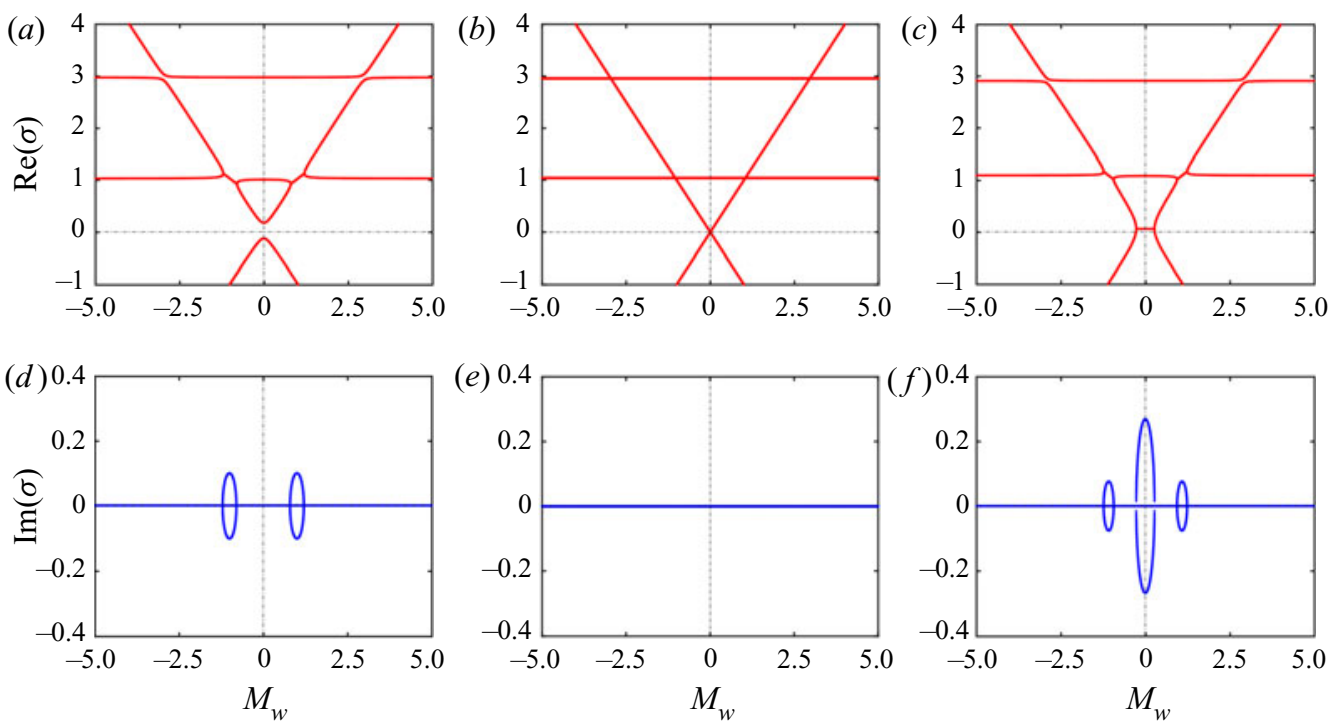

FIGURE 6. Real (upper panels) and imaginary (lower panels) parts of the roots of the dispersion equation (3.30) for $M=M_{0}=2$ and: $(a, d) \beta=0.05$ and $\kappa=\kappa_{0}-0.1,(b, e) \beta=0$ and $\kappa=$ $\kappa_{0} \approx 0.5218134478,(c, f) \beta=0.05$ and $\kappa=\kappa_{0}+0.3$. Notice that the bubbles of instability develop only for $\operatorname{Re}(\sigma)<M_{0}=2$.


FIGURE 7. (a) Stability map of the dispersion equation (3.30) given by its discriminant for $M=M_{0}=2$ and $\beta=0.03$. The regions of real phase speed $\sigma$ are shown in white (stability) and those of the complex $\sigma$ (temporal instability) in blue. The black dotted curves correspond to the approximation (3.36) and the solid red line is the conical approximation (3.43). When $\beta=0$, the blue instability domains degenerate (central) to the ray $\kappa \geq \kappa_{0} \approx 0.5218134478$ and (sides) to the curves (3.39) shown as solid black lines. (b) Stability boundary with the conical singularity at $\kappa=\kappa_{0}, \beta=0$ and $M_{w}=0$, according to (blue, internal surface) the discriminant of the dispersion equation (3.30) and (red, external surface) to the approximation of the cone (3.43).

independent eigenvectors (Kirillov \& Seyranian 2004; Guenther \& Kirillov 2006; Kirillov 2009, 2010, 2013; Kirillov, Guenther \& Stefani 2009).

For this reason, we apply the perturbation theory of double eigenvalues presented in appendix $\mathrm{A}$ to the double zero eigenvalue $\sigma=\sigma_{0}=0$ at the crossing shown in figure $6(b)$ and corresponding to the values of parameters $\beta=\beta_{0}=0, \kappa=\kappa_{0}, M=M_{0}$, 
$M_{w}=M_{w, 0}=0$. A natural extension of the approximation formula (A 18) to the case of four parameters $\beta, \kappa, M_{w}$ and $M$ yields

$$
\begin{aligned}
& (\Delta \sigma)^{2} \frac{1}{2} \partial_{\sigma}^{2} D+\Delta \sigma\left(\partial_{\sigma \beta}^{2} D \Delta \beta+\partial_{\sigma \kappa}^{2} D \Delta \kappa+\partial_{\sigma M}^{2} D \Delta M+\partial_{\sigma M_{w}}^{2} D \Delta M_{w}\right)+\partial_{\beta \kappa}^{2} D \Delta \beta \Delta \kappa \\
& \quad+\frac{1}{2}\left[\partial_{\beta}^{2} D(\Delta \beta)^{2}+\partial_{\kappa}^{2} D(\Delta \kappa)^{2}+\partial_{M}^{2} D(\Delta M)^{2}+\partial_{M_{w}}^{2} D\left(\Delta M_{w}\right)^{2}\right]+\partial_{M \kappa}^{2} D \Delta M \Delta \kappa \\
& \quad+\partial_{M_{w} \kappa}^{2} D \Delta M_{w} \Delta \kappa+\partial_{\beta M}^{2} D \Delta \beta \Delta M+\partial_{\beta M_{w}}^{2} D \Delta \beta \Delta M_{w}+\partial_{\beta M}^{2} D \Delta M_{w} \Delta M \\
& \quad+\partial_{\beta} D \Delta \beta+\partial_{\kappa} D \Delta \kappa+\partial_{M} D \Delta M+\partial_{M_{w}} D \Delta M_{w}=0 .
\end{aligned}
$$

Computing the corresponding partial derivatives of the left part of the dispersion equation (3.30), and evaluating them at $\beta=\beta_{0}=0, \kappa=\kappa_{0}, M=M_{0}, M_{w}=M_{w, 0}=0$, where $M_{0}$ and $\kappa_{0}$ are related by (3.38), we find that the only non-zero derivatives are

$$
\left.\begin{array}{c}
\partial_{\sigma}^{2} D=-\partial_{M_{w}}^{2} D=2 \kappa_{0} M_{0}^{2}-\frac{2}{\kappa_{0} M_{0}^{2}}, \quad \partial_{M \beta}^{2} D=-\partial_{\sigma \beta}^{2} D=2 \kappa_{0} M_{0}, \\
\partial_{\kappa \beta}^{2} D=M_{0}^{4} \kappa_{0}^{2}+M_{0}^{2}-1 .
\end{array}\right\}
$$

Taking this into account in (3.40), we find a simple approximation describing the unfolding of the double zero eigenvalue

$$
\begin{aligned}
& \left(M_{0}^{4} \kappa_{0}^{2}-1\right)\left(\sigma^{2}-M_{w}^{2}\right)-2 \kappa_{0}^{2} M_{0}^{3} \sigma \beta+\kappa_{0} M_{0}^{2}\left(M_{0}^{4} \kappa_{0}^{2}+M_{0}^{2}-1\right)\left(\kappa-\kappa_{0}\right) \beta \\
& \quad+2 \kappa_{0}^{2} M_{0}^{3}\left(M-M_{0}\right) \beta=0 .
\end{aligned}
$$

Let us further assume that $M=M_{0}$ is fixed. Then the last term in (3.42) vanishes, and the discriminant of the resulting quadratic polynomial in $\sigma$ produces the equation of a cone with the apex at $\kappa=\kappa_{0}, M_{w}=0$ and $\beta=0$

$$
M_{0}^{6} \kappa_{0}^{4} \beta^{2}-M_{0}^{2} \kappa_{0}\left(M_{0}^{4} \kappa_{0}^{2}+M_{0}^{2}-1\right)\left(M_{0}^{4} \kappa_{0}^{2}-1\right)\left(\kappa-\kappa_{0}\right) \beta+M_{w}^{2}\left(M_{0}^{4} \kappa_{0}^{2}-1\right)^{2}=0 .
$$

The cone (3.43) is shown in red in figure $7(b)$. With $\beta=0.03, M_{0}=2$ and $\kappa_{0}$ computed by means of (3.38), the approximation (3.43) fits the boundary of the exact instability domain with remarkable precision, as is evident in figure 7(a).

It is easy to see that in the plane $M_{w}=0$ the cone (3.43) defines the two lines

$$
\beta=\frac{\left(M_{0}^{4} \kappa_{0}^{2}-1\right)\left(M_{0}^{4} \kappa_{0}^{2}+M_{0}^{2}-1\right)}{M_{0}^{4} \kappa_{0}^{3}}\left(\kappa-\kappa_{0}\right), \quad \beta=0,
$$

that approximate the instability domain near $\kappa=\kappa_{0}$, see figure 8(b). As soon as $M_{w}$ deviates from zero, the cone (3.43) again provides a very good fit to the actual stability boundary, figure $8(c)$. In the plane

$$
\beta=\frac{\left(M_{0}^{4} \kappa_{0}^{2}-1\right)\left(M_{0}^{4} \kappa_{0}^{2}+M_{0}^{2}-1\right)}{2 M_{0}^{4} \kappa_{0}^{3}}\left(\kappa-\kappa_{0}\right),
$$

the cross-section of the cone (3.43) is described by the two lines

$$
\kappa=\kappa_{0} \pm M_{w} \frac{2 \kappa_{0} M_{0}}{M_{0}^{4} \kappa_{0}^{2}+M_{0}^{2}-1},
$$

that constitute a linear approximation to the stability boundary shown in figure $8(a)$. 

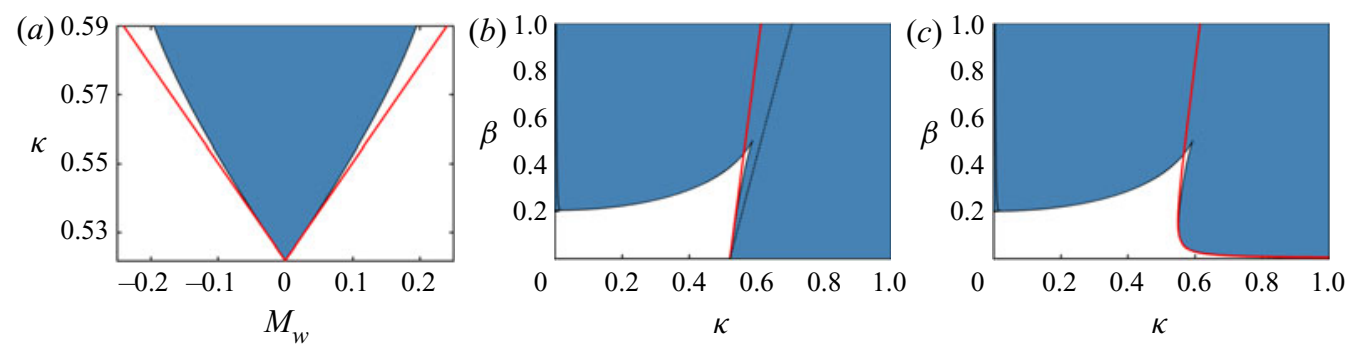

FIGURE 8. For $M=M_{0}=2(a)$ cross-section of the instability domain with the conical singularity shown in figure $7(b)$ in the plane (3.45). The regions of real phase speed $\sigma$ are shown in white (stability) and those of the complex $\sigma$ (temporal instability) in blue. The red lines crossing at the apex of the cone at $\kappa=\kappa_{0} \approx 0.5218134478$ are linear approximations given by (3.46). (b) Cross-section in the plane $M_{w}=0$ of the instability domain and (red line) its linear approximation (3.44) at the conical point $\kappa=\kappa_{0}$. The black dotted line is given by (3.45). (c) Similar cross-section in the plane $M_{w}=0.1$ where the red curve is the approximation (3.43).

\subsubsection{Wave energy of the Nemtsov system for membrane of infinite chord length}

Let us use physical considerations to derive the expression for the averaged over the wave period energy of the Nemtsov system with the membrane of infinite chord length, by combining the approaches of the works by Maissa et al. (2016) and Schulkes et al. (1987).

In the linear wave theory, the energy is a function of the squared wave amplitude (Maissa et al. 2016). Therefore, the total energy per surface area of the membrane resulting both from the wave velocity of the structure and the elastic energy due to its tension is

$$
\mathcal{E}_{m}=\mathcal{K}_{m}+\mathcal{P}_{m}=\frac{1}{2}\left(\operatorname{Re}\left[\partial_{\tau} \xi(x, \tau)\right]\right)^{2}+\frac{1}{2} M_{w}^{2}\left(\operatorname{Re}\left[\partial_{x} \xi(x, \tau)\right]\right)^{2},
$$

where Re stands for the real part of the vibration amplitude that is complex valued because of the assumed plane wave solution

$$
[\phi(x, z, \tau), \eta(x, \tau), \xi(x, \tau)] \sim[\hat{\phi}(z), \hat{\eta}, \hat{\xi}] \mathrm{e}^{\mathrm{i}(\kappa x-\omega \tau)} .
$$

Recall that $\hat{\phi}(z)$ is determined by the expression (3.4) with the coefficients (3.11) and $\hat{\eta}, \hat{\xi}$ are, respectively, displacement amplitudes of the free surface and the membrane.

The energy of the fluid depends on whether we assume a vacuum below the membrane (Nemtsov 1985) or a quiescent medium of the same density as the fluid above the membrane and with a pressure equal to the pressure of the unperturbed fluid (Vedeneev 2004, 2016). The gravitational potential energy of the free surface is the only term contributing to the total potential energy of the fluid in the latter context. Therefore,

$$
\mathcal{P}_{f}=\frac{1}{2} \alpha(\operatorname{Re}[\eta(x, \tau)])^{2} .
$$

The kinetic energy of the flow per unit area is determined by the velocity field $\boldsymbol{u}=$ $\nabla \phi+M e_{x}$, where $\boldsymbol{u}=\boldsymbol{v} / \sqrt{g H}$, that needs to be directly integrated within the limits given 
by the surface of the membrane and the free surface of the fluid,

$$
\begin{aligned}
\mathcal{K}_{f}=\frac{1}{2} \alpha \int_{\operatorname{Re} \xi}^{\operatorname{Re} \eta}\|\operatorname{Re}(\boldsymbol{u})\|^{2} \mathrm{~d} z= & \frac{1}{2} \alpha \int_{\operatorname{Re} \xi}^{\operatorname{Re} \eta}\left[(\operatorname{Re}[\nabla \phi])^{2}+2 M \operatorname{Re}\left[\partial_{x} \phi\right]+M^{2}\right] \mathrm{d} z \\
= & \frac{1}{2} \alpha \int_{\operatorname{Re} \xi}^{\operatorname{Re} \eta}\left[\left(\operatorname{Re}\left[\partial_{x} \phi\right]\right)^{2}+\left(\operatorname{Re}\left[\partial_{z} \phi\right]\right)^{2}\right] \mathrm{d} z \\
& +\alpha M \int_{\operatorname{Re} \xi}^{\operatorname{Re} \eta}\left[\operatorname{Re}\left[\partial_{x} \phi\right]+\frac{M}{2}\right] \mathrm{d} z .
\end{aligned}
$$

From assumption (3.48) and the explicit form of the complex amplitude $\hat{\phi}(z)$ determined by (3.4) with the coefficients (3.11), it follows that

$$
\left.\begin{array}{cl}
\operatorname{Re}\left[\partial_{x} \phi\right]=\mathrm{i} \kappa \hat{\phi}(z) \cos (\kappa x-\omega \tau), & \operatorname{Re}\left[\partial_{z} \phi\right]=\mathrm{i} \partial_{z} \hat{\phi}(z) \sin (\kappa x-\omega \tau), \\
\operatorname{Re} \xi=\hat{\xi} \cos (\kappa x-\omega \tau), & \operatorname{Re} \eta=\hat{\eta} \cos (\kappa x-\omega \tau) .
\end{array}\right\}
$$

Taking into account the expressions (3.51) in (3.50), we find

$$
\int_{\operatorname{Re} \xi}^{\operatorname{Re} \eta}\left(\operatorname{Re}\left[\partial_{x} \phi\right]\right)^{2} \mathrm{~d} z=-\kappa^{2} \cos ^{2}(\kappa x-\omega \tau) \int_{\operatorname{Re} \xi}^{\operatorname{Re} \eta} \hat{\phi}(z)^{2} \mathrm{~d} z .
$$

Similarly, with the help of integration by parts, the Laplace equation (3.3), and expressions (3.51), we obtain

$$
\begin{aligned}
\int_{\operatorname{Re} \xi}^{\operatorname{Re} \eta}\left(\operatorname{Re}\left[\partial_{z} \phi\right]\right)^{2} \mathrm{~d} z & =-\sin ^{2}(\kappa x-\omega \tau)\left\{\left[\hat{\phi} \partial_{z} \hat{\phi}\right]_{\operatorname{Re} \xi}^{\operatorname{Re} \eta}-\int_{\operatorname{Re} \xi}^{\operatorname{Re} \eta} \hat{\phi}\left(\partial_{z}^{2} \hat{\phi}\right) \mathrm{d} z\right\} \\
& =-\sin ^{2}(\kappa x-\omega \tau)\left\{\left[\hat{\phi} \partial_{z} \hat{\phi}\right]_{\operatorname{Re} \xi}^{\operatorname{Re} \eta}-\kappa^{2} \int_{\operatorname{Re} \xi}^{\operatorname{Re} \eta} \hat{\phi}(z)^{2} \mathrm{~d} z\right\} .
\end{aligned}
$$

Finally, following Maissa et al. (2016), we evaluate the last integral term in (3.50) with the help of the Lagrange mean value theorem, which is justified by the assumption that $\eta$ and $\xi$ are infinitesimally small perturbations of the surface boundaries $\partial \Omega_{0}$ and $\partial \Omega_{1}$. Performing this procedure, and then taking into account expressions (3.51), we obtain

$$
\begin{aligned}
& \int_{\operatorname{Re} \xi}^{\operatorname{Re} \eta}\left[\operatorname{Re}\left[\partial_{x} \phi\right]+\frac{M}{2}\right] \mathrm{d} z \\
& \quad=\int_{\operatorname{Re} \xi}^{0} \operatorname{Re}\left[\partial_{x} \phi\right] \mathrm{d} z+\int_{0}^{1} \operatorname{Re}\left[\partial_{x} \phi\right] \mathrm{d} z+\int_{1}^{\operatorname{Re} \eta} \operatorname{Re}\left[\partial_{x} \phi\right] \mathrm{d} z+\frac{1}{2} \int_{\operatorname{Re} \xi}^{\operatorname{Re} \eta} M \mathrm{~d} z \\
& \quad=\left.\operatorname{Re} \eta \operatorname{Re}\left[\partial_{x} \phi\right]\right|_{z=1}-\left.\operatorname{Re} \xi \operatorname{Re}\left[\partial_{x} \phi\right]\right|_{z=0}+\int_{0}^{1} \operatorname{Re}\left[\partial_{x} \phi\right] \mathrm{d} z+\frac{M}{2} \operatorname{Re}(\eta-\xi) \\
& \quad=\mathrm{i} \kappa[\hat{\eta} \hat{\phi}(1)-\hat{\xi} \hat{\phi}(0)] \cos ^{2}(\kappa x-\omega \tau)+\left[\mathrm{i} \kappa \int_{0}^{1} \hat{\phi}(z) \mathrm{d} z+\frac{M}{2}(\hat{\eta}-\hat{\xi})\right] \cos (\kappa x-\omega \tau) .
\end{aligned}
$$

Note that the right-hand sides in the expressions (3.52)-(3.54) are $T$-periodic functions of time, where $T=2 \pi / \omega$. Averaging these expressions over the wave period $T$ according to 
the rule

$$
\langle f(\tau)\rangle=\frac{1}{T} \int_{0}^{T} f(\tau) \mathrm{d} \tau,
$$

we deduce the mean kinetic energy of the fluid

$$
\left\langle\mathcal{K}_{f}\right\rangle=\frac{1}{4} \alpha\left\{-\left[\hat{\phi} \partial_{z} \hat{\phi}\right]_{\partial \Omega_{1}}^{\partial \Omega_{0}}+2 \mathrm{i} \kappa M[\hat{\eta} \hat{\phi}(1)-\hat{\xi} \hat{\phi}(0)]\right\}
$$

The term $\hat{\phi} \partial_{z} \hat{\phi}$ in (3.56) is evaluated with the help of the Bernoulli principle (2.11) and the free surface kinematic condition (2.10) at $\partial \Omega_{0}$, and the wave equation $(2.22 e)$ with the impermeability condition $(2.22 c)$ at $\partial \Omega_{1}$. This yields, respectively,

$$
\left.\begin{array}{c}
\left.\hat{\phi}\right|_{\partial \Omega_{0}}=\frac{\hat{\eta}}{\mathrm{i}(\omega-\kappa M)},\left.\quad \hat{\phi}\right|_{\partial \Omega_{1}}=\frac{\omega^{2}-\kappa^{2} M_{w}^{2}}{\mathrm{i} \alpha(\omega-\kappa M)} \hat{\xi}, \\
\left.\hat{\phi}\right|_{\partial \Omega_{0}}=-\mathrm{i}(\omega-\kappa M) \hat{\eta},\left.\quad \partial_{z} \hat{\phi}\right|_{\partial \Omega_{1}}=-\mathrm{i}(\omega-\kappa M) \hat{\xi} .
\end{array}\right\}
$$

Substituting expressions (3.57) into (3.56) we obtain the final expression for the mean kinetic energy of the fluid

$$
\left\langle\mathcal{K}_{f}\right\rangle=\frac{1}{4}\left\{\alpha \hat{\eta}^{2}-\left(\omega^{2}-\kappa^{2} M_{w}^{2}\right) \hat{\xi}^{2}+2 \mathrm{i} \alpha \kappa M[\hat{\eta} \hat{\phi}(1)-\hat{\xi} \hat{\phi}(0)]\right\}
$$

The other energies of the system, after taking into account (3.51) and time averaging (3.55), become

$$
\left\langle\mathcal{P}_{m}\right\rangle=\frac{1}{4} \kappa^{2} M_{w}^{2} \hat{\xi}^{2}, \quad\left\langle\mathcal{K}_{m}\right\rangle=\frac{1}{4} \omega^{2} \hat{\xi}^{2}, \quad\left\langle\mathcal{P}_{f}\right\rangle=\frac{1}{4} \alpha \hat{\eta}^{2}
$$

Notice that in the absence of the background flow $(M=0)$ the system respects the equipartition of energy

$$
\left\langle\mathcal{P}_{f}\right\rangle+\left\langle\mathcal{P}_{m}\right\rangle=\left\langle\mathcal{K}_{f}\right\rangle+\left\langle\mathcal{K}_{m}\right\rangle
$$

in accordance with the virial theorem (Landau \& Lifschitz 1987), because the flow is irrotational and thus derived from a fluid potential (Schulkes et al. 1987).

After summing up all the different terms given by (3.58) and $(3.59 a-c)$ we obtain the total averaged energy

$$
\langle\mathcal{E}\rangle=\frac{1}{2}\left\{\kappa^{2} M_{w}^{2} \hat{\xi}^{2}+\alpha \hat{\eta}^{2}+\mathrm{i} \alpha \kappa M[\hat{\eta} \hat{\phi}(1)-\hat{\xi} \hat{\phi}(0)]\right\},
$$

thus providing an extension to the case when the velocity field contains a background flow $(M \neq 0)$.

A more suitable expression for the mean total energy can be obtained by expressing the different amplitudes of the system in (3.61) in terms of a unique one, for instance, $\hat{\xi}$. From the kinematic condition (2.10) on the free surface with the plane wave solution (3.48) and 
the coefficients (3.11), it is straightforward to express the surface amplitude $\hat{\eta}$ as

$$
\hat{\eta}=\frac{\mathrm{i} \kappa}{\omega-\kappa M}\left[A \mathrm{e}^{\kappa}-B \mathrm{e}^{-\kappa}\right]=\frac{(\omega-\kappa M)^{2} \hat{\xi}}{(\omega-\kappa M)^{2} \cosh \kappa-\kappa \sinh \kappa} .
$$

Substituting (3.62) into (3.61) and using the complex amplitude $\hat{\phi}(z)$ recovered from the boundary value problem (3.16), we find

$$
\begin{aligned}
\langle\mathcal{E}\rangle= & \frac{\hat{\xi}^{2}}{2}\left\{\kappa^{2} M_{w}^{2}+\alpha \frac{(\omega-\kappa M)^{4}\left(1-(\tanh \kappa)^{2}\right)}{\left[(\omega-\kappa M)^{2}-\kappa \tanh \kappa\right]^{2}}\right. \\
& \left.+\alpha M(\omega-\kappa M) \frac{\left[(\omega-\kappa M)^{4}+\kappa^{2}\right] \tanh \kappa-2 \kappa(\omega-\kappa M)^{2}(\tanh \kappa)^{2}}{\left.\left[(\omega-\kappa M)^{2}-\kappa \tanh \kappa\right)\right]^{2}}\right\} .
\end{aligned}
$$

Next, expressing the term $\kappa^{2} M_{w}^{2}$ by means of the dispersion relation (3.20) and substituting the result into (3.63) yields a more compact formula for the total energy

$$
\langle\mathcal{E}\rangle=\frac{1}{4} \omega\left\{2 \omega+\frac{\alpha}{\kappa} \frac{2(\omega-\kappa M) \tanh \kappa\left[(\omega-\kappa M)^{4}+\kappa^{2}-2 \kappa(\omega-\kappa M)^{2} \tanh \kappa\right]}{\left[(\omega-\kappa M)^{2}-\kappa \tanh \kappa\right]^{2}}\right\} \hat{\xi}^{2} .
$$

Notice that the term in the braces in (3.64) is nothing else but the partial derivative $\partial \mathcal{D} / \partial \omega$ of the dispersion relation (3.20) written in the following equivalent form

$$
\mathcal{D}(\omega, \kappa):=\mathcal{D}_{m}(\omega, \kappa)+\frac{\alpha}{\kappa} \frac{(\omega-\kappa M)^{2}\left[(\omega-\kappa M)^{2} \tanh \kappa-\kappa\right]}{\mathcal{D}_{f}(\omega, \kappa)}=0,
$$

where $\mathcal{D}_{m}=\omega^{2}-\kappa^{2} M_{w}^{2}$ and $\mathcal{D}_{f}=\left[(\omega-\kappa M)^{2}-\kappa \tanh \kappa\right]$ stand for the dispersion relation of, respectively, the free membrane and the free surface flow with a rigid boundary at the bottom. This proves that our total energy per unit area, averaged over the wave period, possesses the following simple representation in terms of the dispersion relation

$$
\langle\mathcal{E}\rangle=\frac{1}{4} \omega \frac{\partial \mathcal{D}}{\partial \omega} \hat{\xi}^{2} .
$$

The representation (3.66) can be found, e.g. in Cairns (1979), and can be derived in the frame of the general Lagrangian variational approach (Ostrovskii, Rybak \& Tsimring 1986; Whitham 1999), see also the recent work by Fukumoto, Hirota \& Mie (2014) for historical notes and application to stability of vortices. Notice that according to (3.66) the energy vanishes at the points where $\omega=0$ or $\partial \mathcal{D} / \partial \omega=0$, the latter condition corresponding to the existence of multiple roots of the dispersion relation. Correspondingly, the ratio $\langle\mathcal{E}\rangle / \omega$, which is the averaged wave action $\langle\mathcal{A}\rangle$ (Whitham 1999; Zhang et al. 2016), vanishes only at the locations of the multiple eigenvalues, cf. figures 2 and 9. In the latter figure as well as in figure 10 we show several computations of the averaged wave energy and wave action over the fluid Mach number $M$, and, respectively, the membrane Mach number $M_{w}$, where $\omega$ is calculated with the use of the dispersion relation (3.20). 
(a)

$\langle\mathcal{E}\rangle$



(e)

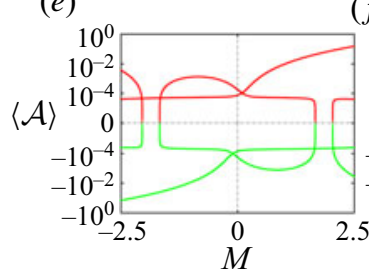

(b)

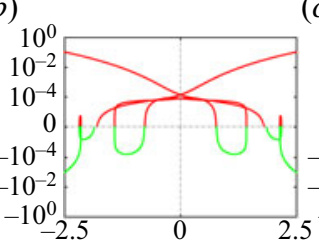

(c)

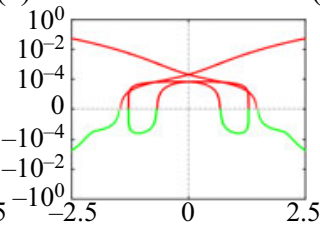

(d)

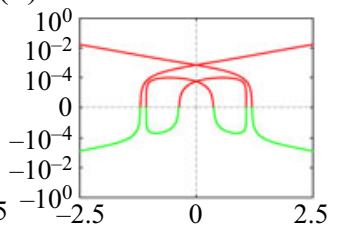

(f) $(g)$

g) $(h)$

(h) ${ }_{10}$
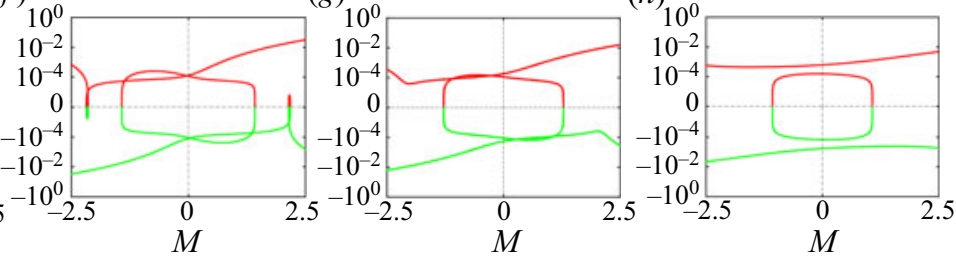

FIGURE 9. The averaged wave energy $(a-d)\langle\mathcal{E}\rangle$ given by the expression (3.64) and the action $(e-h)\langle\mathcal{A}\rangle=\langle\mathcal{E}\rangle / \omega$ over the Mach number $M$ evaluated for $M_{w}=1, \kappa=1, \hat{\xi}=0.01$ and: $(a, e) \alpha=0.1,(b, f) \alpha=0.5,(c, g) \alpha=1$ and $(d, h) \alpha=5$. Positive (respectively negative) energy/action is represented in red (respectively green).

(a)

$\langle\mathcal{E}\rangle$

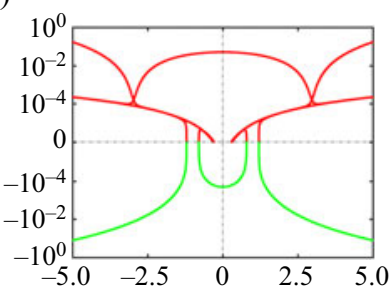

(d)

$\langle\mathcal{A}\rangle$



(b)

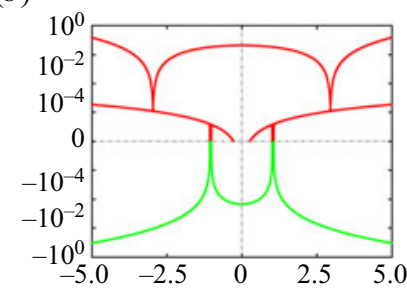

(e)

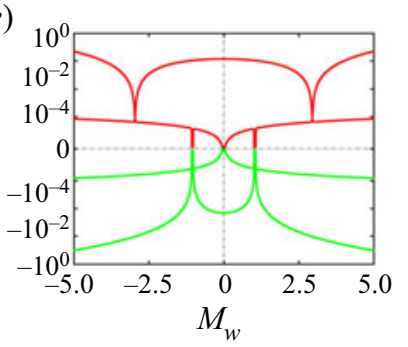

(c)

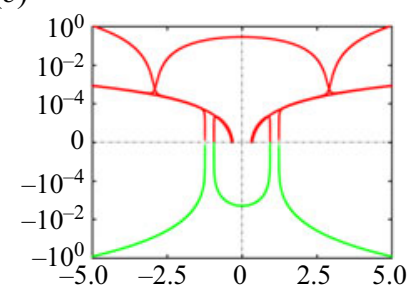

$(f)$

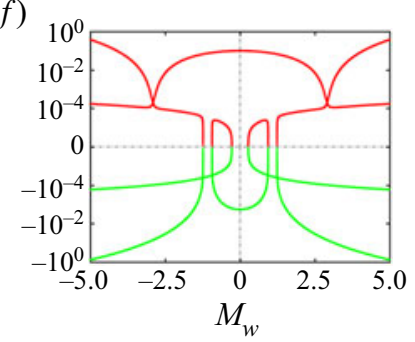

FIGURE 10. The averaged wave energy (upper panels) $\langle\mathcal{E}\rangle$ given by the expression (3.64) and the action (lower panels) $\langle\mathcal{A}\rangle=\langle\mathcal{E}\rangle / \omega$ over the Mach number $M_{w}$ for $\hat{\xi}=0.01, M=M_{0}=2$, and $(a, d) \quad \beta=0.05$ and $\kappa=\kappa_{0}-0.1,(b, e) \beta=10^{-3}$ and $\kappa=\kappa_{0} \approx 0.5218134478,(c, f)$ $\beta=0.05$ and $\kappa=\kappa_{0}+0.3$.

\section{Discussion}

Comparing the eigenvalue plots of figures 2 and 6 with the averaged wave energy and wave action of each branch that are shown in figures 9 and 10, respectively, we notice that flutter instability is necessarily accompanied with the interaction of waves of opposite sign of energy/action. In contrast to the action, the energy changes sign also at the points where the phase velocity $\sigma$ changes sign, quite in accordance with (3.66).

Looking now at the roots $(3.28 a, b)$ of the decoupled dispersion equation (3.26), we conclude that the elastic waves $\sigma_{1}^{ \pm}= \pm M_{w}$ propagating in the membrane always have 
positive energy whereas among the surface gravity waves $\sigma_{2}^{ \pm}=M \pm \sqrt{(\tanh \kappa) / \kappa}$ it is the energy of the slow wave $\sigma_{2}^{-}$that becomes negative for $M>0$ as soon as $M>$ $\sqrt{(\tanh \kappa) / \kappa}$. Therefore at the crossing (3.29) corresponding to $M_{0}^{+}=M_{w}+\sqrt{(\tanh \kappa) / \kappa}$ the positive energy/action elastic wave meets the slow surface gravity wave that carries negative energy/action (Nemtsov 1985).

With $\beta$ increasing from zero, the crossing unfolds causing the eigenvalue branches to merge on the interval bounded by the points where $\partial_{\omega} D=0$. At these exceptional points (Kirillov 2013) both the energy and the action change sign, see figures 9 and 10. On the interval the roots are complex and form the bubble of instability, see figures 2 and 6 .

Since the fast surface gravity wave carries positive energy, one needs to add energy to the flow in order to excite this wave on the flow. In contrast, in order for the slow surface gravity wave carrying negative energy to build up on the flow, the energy must be extracted from the flow (Nezlin 1976) via some mechanism for dissipation of its energy. In the Nemtsov problem, such a mechanism is the energy transfer from the slow surface gravity wave to an elastic wave associated with the membrane, which is a stationary medium and therefore has positive energy (Nezlin 1976). One can say that this transferred energy yields flutter of the membrane due to emission of the slow surface gravity wave carrying negative energy.

In figures 2 and 6 as well as in figures 9 and 10 we observe that the flutter instability of the membrane occurs only if the velocity of the flow is higher than the phase velocity of the oscillations on the surface of the flow, $\sigma<M$, i.e. the flow moves faster than the waves it can excite (Nezlin 1976; Nemtsov \& Eidman 1987). The condition $\omega=M \kappa$ or $\sigma=M$ is known as the Cerenkov condition for emission of radiation by a moving source (Ginzburg \& Frank 1947; Nezlin 1976; Ginzburg 1996; Bekenstein \& Schiffer 1998; Carusotto \& Rousseaux 2013). Substituted into a dispersion relation, the Cerenkov condition transforms the former into an expression defining a surface in the space of wavenumbers that determines the wake pattern behind the source (Schulkes et al. 1987; Carusotto \& Rousseaux 2013). For the supercritical velocities $M>\sigma$ the surface in the space of wavenumbers develops a conical singularity known as the Cerenkov cone (Nemtsov 1985; Carusotto \& Rousseaux 2013) with the angular aperture

$$
2 \arccos \left(\frac{\sigma}{M}\right) \text {. }
$$

The anomalous Doppler effect (ADE) is the change in the sign of the field frequency radiated into the Cerenkov cone as compared with the field radiated outside this cone (Nezlin 1976; Gaponov-Grekhov et al. 1983; Nemtsov 1985; Abramovich et al. 1986; Carusotto \& Rousseaux 2013). It is exactly the slow surface gravity wave that satisfies this condition

$$
\sigma_{2}^{-}-M=-\sqrt{\frac{\tanh \kappa}{\kappa}}<0 .
$$

Hence, for the one-dimensional or, more precisely, plane two-dimensional waves, both the negative energy waves and the ADE correspond simply to waves with phase velocity lower than the flow velocity and wave vector pointing in the same direction as the flow (Nemtsov 1985; Ostrovskii et al. 1986). In our case, the radiated slow gravity wave increases the energy of oscillations of the membrane at the expense of the energy of the flow that supports this wave.

Finally, we plot the dispersion curves $\omega(\kappa)$ in figure 11, which show that the slow surface gravity wave branch and the membrane branch interact under the line $\operatorname{Re}(\omega)=\kappa M$, if $\kappa>0$. Substituting the Cerenkov condition in the dispersion relation (3.20) we reduce 


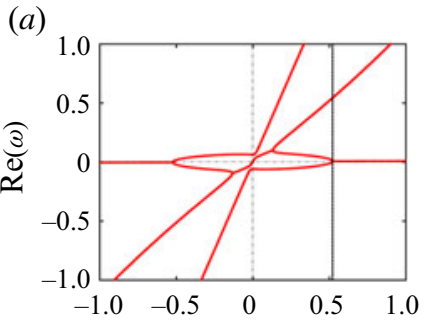

(d)
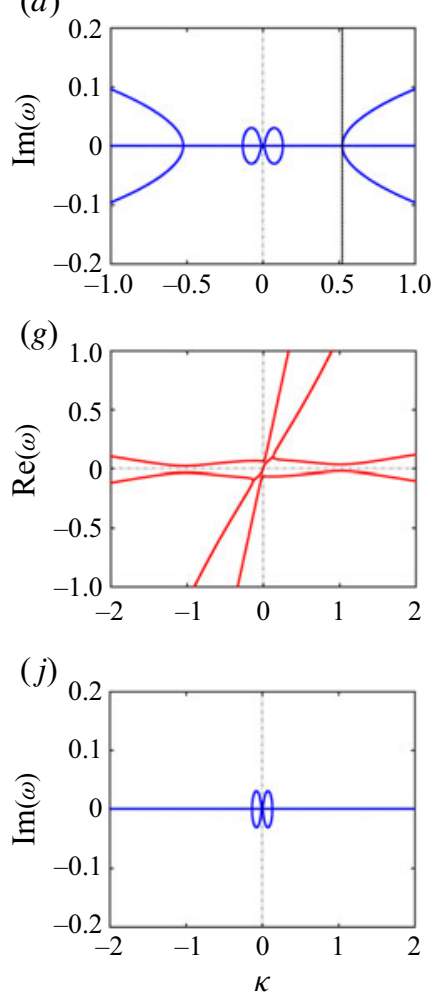

(b)

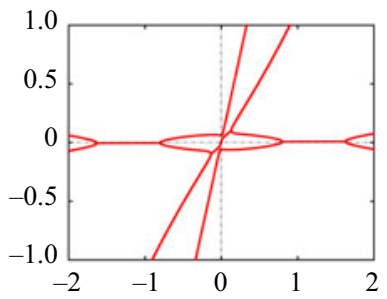

(e)

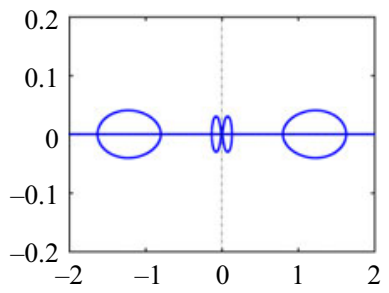

$(h)$

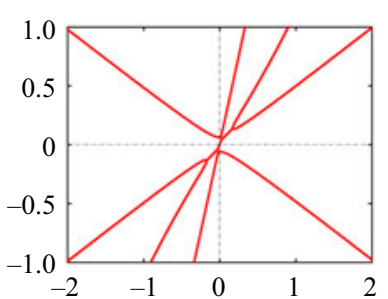

$(k)$

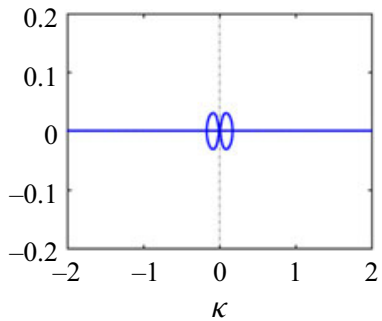

(c)

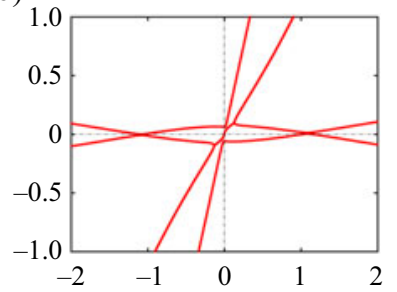

(f)

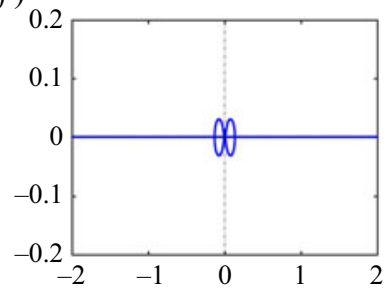

(i)

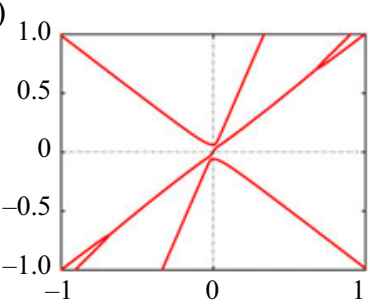

(l)

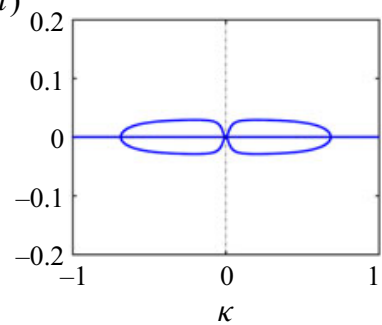

FIGURE 11. Dispersion curves ((red) real and (blue) imaginary parts of the roots $\omega$ of the dispersion relation (3.20)) for $M=2, \alpha \approx 0.0036725648$ and $(a, d) M_{w}=0,(b, e) M_{w}=0.09$, $(c, f) M_{w}=0.0967,(g, j) M_{w}=0.1,(h, k) M_{w}=0.5,(i, l) M_{w}=1$. Vertical dashed lines in the panels $(a, d)$ correspond to $\kappa=\kappa_{0} \approx 0.5218134478$ and mark the onset of instability corresponding to the central instability zone in figure $7(a)$ and the conical instability zone in figure $7(b)$.

it to $\left(M_{w}^{2}-M^{2}\right) \tanh \kappa=0$, thus providing a rationale for the absence of instabilities for $M_{w}^{2}>M^{2}$ that is evident in all our stability diagrams.

\section{Conclusion}

Through the revival of a classical hydrodynamical model performed in this work, we have been able to extend the stability analysis and to enhance knowledge of the underlying physics by making connections with the fundamental concepts such as negative energy waves and the ADE, supported by advanced mathematical tools. 
Our continuation of Nemtsov's investigation of the radiation-induced flutter of a membrane in a uniform flow with the new derivation of the dispersion relation for a fluid layer of arbitrary depth and membrane of infinite chord length has led to a significant improvement in the computation of stability diagrams without any limitation on the range of the system parameters.

An exhaustive stability analysis has been performed using the original perturbation theory of multiple roots of the dispersion relation to compare with the exact stability domains, and both computations are proven to be in excellent agreement. More precisely, the crossings and avoided crossings of the dispersion curves are very well approximated by the simplified expressions for the phase speed of the membrane and fluid modes derived with the perturbation approach.

After computing the discriminant of the full dispersion relation, we have identified a new instability domain arising from a conical singularity in the parameter space that was not present in the early study of Nemtsov. This new domain is associated with a low-frequency flutter for short wavelengths and corresponds to the case when the velocity of propagation of elastic waves in the membrane is much smaller than the velocity of the flow.

Moreover, following the procedures used in previous studies on simplified hydrodynamical systems to calculate the averaged wave energy and after developing the method further to take into account the coupling between the free surface of the flow and the elastic membrane on the bottom, we have obtained an elegant and applicable expression for the total averaged energy. We have verified that, in the absence of the background flow, the system respects the equipartition of energy in accordance with the virial theorem, thus confirming that the existence of the negative energy waves can only be possible when the fluid is in motion.

We have shown that the formula for the total averaged energy recovered in our work by means of the direct integration of different physical fields is expressed via the derivative of the dispersion relation with respect to the frequency of oscillations and reduces exactly to the form described by Cairns (1979).

The ADE is a direct consequence of the relative motion of an oscillator in a medium and more precisely, it occurs when the internal energy of the system increases due to the emission of negative energy waves (NEW). In our context, while the system is composed of a fluid layer and a membrane, such a phenomenon has been proved by Nemtsov to exist only when the conditions of phase synchronism and NEW emission are satisfied. The criterion for the phase synchronism in the system is easily identified in the computations of the dispersion curves as the crossings of the different branches that lead to the onset of positive growth rate and therefore to temporal instability. The latter phenomenon is a natural consequence of the highly excited state of energy that the system transits to due to the dominance of NEW over the waves carrying positive energy. Indeed, NEW emission is known as a process that increases the total energy of an oscillatory system while radiating energy away from the oscillator and, only when this gain in internal energy exceeds the losses from the contribution of positive energy modes, the total energy of the system starts growing in amplitude. Hence, it requires to have waves carrying energy of opposite signs that interact for the instability to develop.

Our expressions for the action and energy of the Nemtsov system demonstrated as expected the collision of waves carrying positive and negative energy as the onset for the radiative instability and the flutter of the membrane. Such a phenomenon is well known in the community of dynamical systems, but in this context, it is associated with the emission of NEW in the region of ADE. Hence, in addition to improving the stability analysis of the Nemtsov system and computing the averaged wave energy, our study provides a further, more detailed, examination of the ADE in hydrodynamics. Despite our problem being 
restricted to the study of planar waves, with the latter being emitted only in the horizontal direction, it is still sufficient for exploring the connection between the ADE and flutter theory.

An extension of this work to the case of a membrane with a chord of finite size, as described by the system of (2.22), is a promising necessary next step requiring asymptotic methods for the global stability analysis and numerical computations that we leave for future work.

\section{Acknowledgements}

We thank an anonymous referee for bringing important early works to our attention. We are grateful to Professor T.J. Bridges for helpful discussions. J.L. was supported by a PhD Scholarship from Northumbria University.

\section{Declaration of interests}

The authors report no conflict of interest.

\section{Appendix A. Sensitivity analysis of dispersion equations}

In contrast to other works on frequency coalescence, e.g. Triantafyllou \& Triantafyllou (1991), we adapt a more systematic multiparameter sensitivity analysis that can be found, e.g. in Kirillov \& Seyranian (2002), Kirillov \& Seyranian (2004), Kirillov (2007a), Kirillov (2007b), Kirillov (2009), Kirillov et al. (2009), Kirillov (2010) and Kirillov (2013).

Let us consider the dispersion equation

$$
D(\omega, p, q)=0
$$

where $D$ is a smooth function of scalar arguments $\omega, p$ and $q$. Assume that $D(\omega)$ is a polynomial of degree $n$ in $\omega$.

\section{A.1. Sensitivity of simple roots}

Let at $p=p_{0}$ and $q=q_{0}$ (A 1$)$ have a simple root $\omega_{0}$ such that

$$
D_{0}:=D\left(\omega_{0}, p_{0}, q_{0}\right)=0
$$

where we use the symbol $:=$ to indicate a definition.

Following Kirillov \& Seyranian (2002), Kirillov \& Seyranian (2004), Kirillov (2007a), Kirillov (2007b), Kirillov (2010) and Kirillov (2013), we assume that $p=p(\varepsilon)$ and $q=$ $q(\varepsilon)$. For $|\varepsilon|$ sufficiently small we can represent these functions as Taylor series

$$
\left.\begin{array}{l}
p(\varepsilon)=p_{0}+\varepsilon \frac{\mathrm{d} p}{\mathrm{~d} \varepsilon}+\frac{\varepsilon^{2}}{2} \frac{\mathrm{d}^{2} p}{\mathrm{~d} \varepsilon^{2}}+o\left(\varepsilon^{2}\right), \\
q(\varepsilon)=q_{0}+\varepsilon \frac{\mathrm{d} q}{\mathrm{~d} \varepsilon}+\frac{\varepsilon^{2}}{2} \frac{\mathrm{d}^{2} q}{\mathrm{~d} \varepsilon^{2}}+o\left(\varepsilon^{2}\right),
\end{array}\right\}
$$

with the derivatives evaluated at $\varepsilon=0$, and $p_{0}:=p(0)$ and $q_{0}:=q(0)$. Then, $\omega=\omega(\varepsilon)$ is also a root of (A 1), i.e. it satisfies the equation

$$
D_{\varepsilon}:=D(\omega(\varepsilon), p(\varepsilon), q(\varepsilon))=0 .
$$


Differentiating (A 4), we find

$$
\frac{\mathrm{d}}{\mathrm{d} \varepsilon} D_{\varepsilon}=\partial_{\omega} D \frac{\mathrm{d} \omega}{\mathrm{d} \varepsilon}+\partial_{p} D \frac{\mathrm{d} p}{\mathrm{~d} \varepsilon}+\partial_{q} D \frac{\mathrm{d} q}{\mathrm{~d} \varepsilon}=0
$$

where the partial derivatives are evaluated at $\omega=\omega_{0}, q=q_{0}, p=p_{0}$.

Denoting $\Delta \omega=\varepsilon(\mathrm{d} \omega / \mathrm{d} \varepsilon) \approx \omega-\omega_{0}, \Delta q=\varepsilon(\mathrm{d} q / \mathrm{d} \varepsilon) \approx q-q_{0}$ and $\Delta p=\varepsilon(\mathrm{d} p / \mathrm{d} \varepsilon) \approx$ $p-p_{0}$, we find the expression for the increment of the simple root $\omega_{0}$ of (A 1) when the parameters depart from the values $q_{0}$ and $p_{0}$

$$
\Delta \omega=-\frac{\partial_{p} D}{\partial_{\omega} D} \Delta p-\frac{\partial_{q} D}{\partial_{\omega} D} \Delta q+o(|\Delta p|,|\Delta q|) .
$$

\section{A.2. Double root of the dispersion relation: generic case}

Let at $p=p_{0}$ and $q=q_{0}$ the dispersion equation (A 1) have a double root $\omega_{0}$, which implies

$$
\left.\begin{array}{c}
D_{0}=0, \\
\partial_{\omega} D_{0}:=\partial_{\omega} D\left(\omega_{0}, p_{0}, q_{0}\right)=0 .
\end{array}\right\}
$$

Assume that the perturbation of the parameters (A 3) causes splitting of the double root $\omega_{0}$ which generically is described by the Newton-Puiseux series (Kirillov \& Seyranian 2002, 2004; Kirillov 2007a,b, 2010, 2013)

$$
\omega(\varepsilon)=\omega_{0}+\omega_{1} \varepsilon^{1 / 2}+\omega_{2} \varepsilon+\omega_{3} \varepsilon^{3 / 2}+\omega_{4} \varepsilon^{2}+o\left(\varepsilon^{2}\right) .
$$

Expanding $D_{\varepsilon}$ as

$$
D_{\varepsilon}=\sum_{s=0}^{n} \frac{\left(\omega(\varepsilon)-\omega_{0}\right)^{s}}{s !}\left(\partial_{\omega}^{s} D+\varepsilon \partial_{\omega}^{s} D_{1}+\varepsilon^{2} \partial_{\omega}^{s} D_{2}+o\left(\varepsilon^{2}\right)\right),
$$

where

$$
\left.\begin{array}{c}
D_{1}:=\partial_{p} D \frac{\mathrm{d} p}{\mathrm{~d} \varepsilon}+\partial_{q} D \frac{\mathrm{d} q}{\mathrm{~d} \varepsilon}, \\
D_{2}:=\frac{1}{2} \partial_{p} D \frac{\mathrm{d}^{2} p}{\mathrm{~d} \varepsilon^{2}}+\frac{1}{2} \partial_{q} D \frac{\mathrm{d}^{2} q}{\mathrm{~d} \varepsilon^{2}}+\frac{1}{2}\left(\partial_{p}^{2} D \frac{\mathrm{d}^{2} p}{\mathrm{~d} \varepsilon^{2}}+2 \partial_{p q}^{2} D \frac{\mathrm{d} p}{\mathrm{~d} \varepsilon} \frac{\mathrm{d} q}{\mathrm{~d} \varepsilon}+\partial_{q}^{2} D \frac{\mathrm{d}^{2} q}{\mathrm{~d} \varepsilon^{2}}\right),
\end{array}\right\}
$$

substituting expansion (A 8) into (A 9) and collecting the coefficients at the same powers of $\varepsilon$, we find

$$
\left.\begin{array}{c}
D_{0}=0, \\
\omega_{1} \partial_{\omega} D_{0}=0, \\
D_{1}+\omega_{1}^{2} \frac{1}{2} \partial_{\omega}^{2} D+\omega_{2} \partial_{\omega} D_{0}=0 .
\end{array}\right\}
$$

Looking for the coefficient $\omega_{1} \neq 0$, we see that the first two equations of (A 11) are satisfied in view of the fact that $\omega_{0}$ is a double root of the dispersion equation (A 1). Taking this 
into account, the last of (A 11) yields the expression for the coefficient $\omega_{1}$ in the expansion (A 8)

$$
\omega_{1}^{2}=-D_{1}\left(\frac{1}{2} \partial_{\omega}^{2} D\right)^{-1},
$$

where all the partial derivatives are calculated at $p=p_{0}, q=q_{0}, \omega=\omega_{0}$.

Therefore, if $D_{1} \neq 0$, the double root $\omega_{0}$ splits under variation of parameters (A 3) according to the formula

$$
\omega=\omega_{0} \pm \sqrt{-\varepsilon D_{1}\left(\frac{1}{2} \partial_{\omega}^{2} D\right)^{-1}}+o\left(|\varepsilon|^{1 / 2}\right) .
$$

In terms of the increments of the parameters, we can re-write (A 13) as

$$
\Delta \omega= \pm \sqrt{-\left(\partial_{p} D \Delta p+\partial_{q} D \Delta q\right)\left(\frac{1}{2} \partial_{\omega}^{2} D\right)^{-1}}+o\left(|\Delta p|^{1 / 2},|\Delta q|^{1 / 2}\right) .
$$

\section{A.3. Double root of the dispersion relation: degenerate case}

The case $D_{1}=0$ is degenerate, because the leading term in (A 13) of order $\varepsilon^{1 / 2}$ vanishes and the expansion (A 8) is no longer valid, see e.g. Kirillov \& Seyranian (2004). Substituting expansion (A 13) with $\omega_{1}=0$ into (A9) and collecting coefficients of the same powers of $\varepsilon$, we obtain

$$
\left.\begin{array}{c}
D_{1}+\omega_{2} \partial_{\omega} D_{0}=0, \\
\omega_{3} \partial_{\omega} D_{0}=0, \\
\partial_{\omega}^{2} D+\omega_{2} \partial_{\omega} D_{1}+\omega_{4} \partial_{\omega} D_{0}=0 .
\end{array}\right\}
$$

Taking into account that $\partial_{\omega} D_{0}=0$ since $\omega_{0}$ is the double root and that $D_{1}=0$ due to our assumption, we conclude that the first two of (A 15) hold automatically. The third one simplifies as follows:

$$
\omega_{2}^{2} \frac{1}{2} \partial_{\omega}^{2} D+\omega_{2} \partial_{\omega} D_{1}+D_{2}=0
$$

where all the derivatives are calculated at $\omega=\omega_{0}, p=p_{0}$ and $q=q_{0}$.

Therefore, the degeneracy, $D_{1}=0$, implies that the double root $\omega_{0}$ splits according to the formula

$$
\omega=\omega_{0}+\omega_{2} \varepsilon+o(\varepsilon)
$$

where the coefficient $\omega_{2}$ is a root of the polynomial (A 16).

In combination with (A 10) and (A 17) the polynomial (A 16) transforms into

$$
\begin{aligned}
& (\Delta \omega)^{2} \frac{1}{2} \partial_{\omega}^{2} D+\Delta \omega\left(\partial_{\omega p}^{2} D \Delta p+\partial_{\omega q}^{2} D \Delta q\right)+\frac{1}{2}\left[\partial_{p}^{2} D(\Delta p)^{2}+2 \partial_{p q}^{2} D \Delta p \Delta q+\partial_{q}^{2} D(\Delta q)^{2}\right] \\
& \quad+\partial_{p} D \Delta p+\partial_{q} D \Delta q=0 .
\end{aligned}
$$

Extension to the case of more than two parameters is straightforward, see e.g. Kirillov \& Seyranian (2002), Kirillov \& Seyranian (2004), Kirillov (2007a), Kirillov (2007b), Kirillov (2009), Kirillov et al. (2009), Kirillov (2010) and Kirillov (2013). 


\section{REFERENCES}

Abramovich, B. S., Mareev, E. A. \& Nemtsov, B. E. 1986 Instability in the oscillations of a moving oscillator while it radiates surface and internal waves. Fluid Dyn. 21 (1), 147-149.

Arzhannikov, A. V. \& Kotelnikov, I. A. 2016 Excitation of ship waves by a submerged object: new solution to the classical problem. Phys. Rev. E 94, 023103.

Banichuk, N., Barsuk, A., Jeronen, J., Tuovinen, T. \& Neittaanmäki, P. 2019 Stability of Axially Moving Materials, Solid Mechanics and Its Applications, vol. 259. Springer.

Banichuk, N., Jeronen, J., NeittaAnmäKi, P. \& TuOvinen, T. 2010 Static instability analysis for travelling membranes and plates interacting with axially moving ideal fluid. J. Fluids Struct. 26, 274-291.

Barbone, P. E. \& Crighton, D. G. 1994 Vibrational modes of submerged elastic bodies. Appl. Acoust. 43, 295-317.

Barcelo, C., Liberati, S. \& Visser, M. 2011 Analogue gravity. Living Rev. Relativ. 14, 3.

Bekenstein, J. D. \& Schiffer, M. 1998 The many faces of superradiance. Phys. Rev. D 58, 064014.

BENJAmin, T. B. 1963 The threefold classification of unstable disturbances in flexible surfaces bounding inviscid flows. J. Fluid Mech. 16, 436-450.

Bochkarev, S. A., Lekomtsev, S. V. \& Matveenko, V. P. 2016 Hydroelastic stability of a rectangular plate interacting with a layer of ideal flowing fluid. Fluid Dyn. 51 (6), 821-833.

Bolotin, V. V. 1963 Nonconservative Problems of the Theory of Elastic Stability. Pergamon Press.

Brito, R., CARdoso, V. \& PANI, P. 2015 Superradiance, Lecture Notes in Physics, vol. 906. Springer.

CAIRns, R. A. 1979 The role of negative energy waves in some instabilities of parallel flows. J. Fluid Mech. 92, 1-14.

Carusotto, I. \& Rousseaux, G. 2013 The Cerenkov effect revisited: from swimming ducks to zero modes in gravitational analogues. In Analogue Gravity Phenomenology (ed. D. Faccio, F. Belgiorno, S. Cacciatori, V. Gorini, S. Liberati \& U. Moschella), Lecture Notes in Physics, vol. 870. Springer.

Das, S., Kar, P., SAhoo, T. \& MeYlan, M. H. 2018 Flexural-gravity wave motion in the presence of shear current: wave blocking and negative energy waves. Phys. Fluids 30, 106606.

Das, S., Sahoo, T. \& Meylan, M. H. 2018a Dynamics of flexural gravity waves: from sea ice to Hawking radiation and analogue gravity. Proc. R. Soc. A 474, 20170223.

Das, S., Sahoo, T. \& MeYlan, M. H. $2018 b$ Flexural-gravity wave dynamics in two-layer fluid: blocking and dead water analogue. J. Fluid Mech. 854, 121-145.

DOARÉ, O. \& DE LANGRE, E. 2006 The role of boundary conditions in the instability of one-dimensional systems. Eur. J. Mech. (B/Fluids) 25, 948-959.

DowelL, E. H. 1966 Flutter of infinitely long plates and shells. Part 1. Plate. AIAA J. 4, 1370-1377.

Dowell, E. H. 2015 A Modern Course in Aeroelasticity, 5th Revised and Enlarged edn. Solid Mechanics and Its Applications, vol. 217. Springer.

Dowell, E. H. \& Ventres, C. S. 1970 On the flutter of low aspect ratio plates. AIAA J. 8, 1162-1164.

Dugundi, J., Dowell, E. \& Perkin, B. 1963 Subsonic flutter of panels on a continuous elastic foundation. AIAA J. 1, 1146-1154.

Faltinsen, O. M. \& Semenov, Y. A. 2008 The effect of gravity and cavitation on a hydrofoil near the free surface. J. Fluid Mech. 597, 371-394.

Fukumoto, Y., Hirota, M. \& Mie, Y. 2014 Representation of wave energy of a rotating flow in terms of the dispersion relation. In Nonlinear Physical Systems: Spectral Analysis, Stability and Bifurcations (ed. O. N. Kirillov \& D. E. Pelinovsky). Wiley-ISTE.

Gaponov-Grekhov, A. V., Dolina, I. S. \& Ostrovskit, L. A. 1983 The anomalous Doppler effect and the radiation instability of oscillator motion in hydrodynamics. Dokl. Akad. Nauk SSSR 268 (4), 827-831. In Russian.

GINZBURG, V. L. 1996 Radiation by uniformly moving sources (Vavilov-Cherenkov effect, transition radiation, and other phenomena). Phys.-Uspekhi 39 (10), 973-982.

GinZburG, V. L. \& Frank, I. M. 1947 On the Doppler effect at the superluminal velocity. Dokl. Akad. Nauk SSSR 56, 583-586.

GISLASON, T. JR. 1971 An experimental investigation of panel divergence at subsonic speeds. AIAA J. 9, 2252-2258. 
Goland, M. \& LuKe, Y. L. 1954 An exact solution for two-dimensional linear panel flutter at supersonic speeds. J. Aeronaut. Sci. 21 (4), 275-276.

Greenhill, A. G. 1886 Wave motion in hydrodynamics. Am. J. Maths 9 (1), 62-96.

GUENTHER, U. \& KIRILLOV, O. N. 2006 A Krein space related perturbation theory for MHD $\alpha^{2}$-dynamos and resonant unfolding of diabolical points. J. Phys. A: Math. Gen. 39, 10057-10076.

Hagerty, P., Bloch, A. M. \& Weinstein, M. I. 2003 Radiation induced instability. SIAM J. Appl. Math. 64 (2), 484-524.

KiRILlov, O. N. 2007a Bifurcation of the roots of the characteristic polynomial and destabilization paradox in friction induced oscillations. Theor. Appl. Mech. 34 (2), 87-109.

Kirillov, O. N. $2007 \mathrm{~b}$ On the stability of nonconservative systems with small dissipation. J. Math. Sci. 145 (5), 5260-5270.

Kirillov, O. N. 2009 Campbell diagrams of weakly anisotropic flexible rotors. Proc. R. Soc. A 465 (2109), 2703-2723.

KIRILlov, O. N. 2010 Eigenvalue bifurcation in multiparameter families of non-self-adjoint operator matrices. Z. Angew. Math. Phys. 61 (2), 221-234.

Kirillov, O. N. 2013 Nonconservative Stability Problems of Modern Physics, De Gruyter Studies in Mathematical Physics, vol. 14. De Gruyter.

Kirillov, O. N., Guenther, U. \& Stefani, F. 2009 Determining role of Krein signature for three dimensional Arnold tongues of oscillatory dynamos. Phys. Rev. E 79 (1), 016205.

Kirillov, O. N. \& Seyranian, A. P. 2002 Metamorphoses of characteristic curves in circulatory systems. J. Appl. Math. Mech. 66 (3), 371-385.

Kirillov, O. N. \& Seyranian, A. P. 2004 Collapse of the Keldysh chains and stability of continuous non-conservative systems. SIAM J. Appl. Math. 64 (4), 1383-1407.

Kornecki, A., Dowell, E. H. \& O'Brien, J. 1976 On the aeroelastic instability of two-dimensional panels in uniform incompressible flow. J. Sound Vibr. 47, 163-178.

LAMB, H. 1900 On a peculiarity of the wave-system due to the free vibrations of a nucleus in an extended medium. Proc. Lond. Math. Soc. s1-32 (1), 208-213.

Landau, L. D. \& Lifschitz, E. M. 1987 Fluid Mechanics, 2nd edn. Pergamon Press.

Maissa, P., Rousseaux, G. \& Stepanyants, Y. 2016 Negative energy waves in a shear flow with a linear profile. Eur. J. Mech. (B/Fluids) 56, 192-199.

Mavroyiakoumou, C. \& Alben, S. 2020 Large-amplitude membrane flutter in inviscid flow. J. Fluid Mech. 891, A23-1-24.

Miles, J. W. 1947 The aerodynamic forces on an oscillating air foil at supersonic speeds. J. Aeronaut. Sci. 14 (6), 351-358.

MiLeS, J. W. 1956 On the aerodynamic stability of thin panels. J. Aeronaut. Sci. 23, 771-780.

MinAmi, H. 1998 Added mass of a membrane vibrating at finite amplitude. J. Fluids Struct. 12, 919-932.

Mohapatra, S. C. \& SAHOo, T. 2011 Surface gravity wave interaction with elastic bottom. Appl. Ocean Res. 33, 31-40.

Nemtsov, B. E. 1985 Flutter effect and emission in the region of anomalous and normal Doppler effects. Radiophys. Quantum Electron. 28 (12), 1076-1079.

Nemtsov, B. E. \& Eidman, V. Y. 1987 Hyperlight biresonant radiation (review). Radiophys. Quantum Electron. 30, 171-188.

Newman, B. G. \& Paidoussis, M. P. 1991 The stability of two-dimensional membranes in streaming flow. J. Fluids Struct. 5, 443-454.

NeZlin, M. V. 1976 Negative-energy waves and the anomalous Doppler effect. Sov. Phys. Uspekhi 19, 946-954.

Ostrovskit, L. A., RYbaK, S. A. \& Tsimring, L. Sh. 1986 Negative energy waves in hydrodynamics. Sov. Phys. Uspekhi 29 (11), 1040-1052.

Robertson, S. \& Rousseaux, G. 2018 Viscous dissipation of surface waves and its relevance to analogue gravity experiments. arXiv: $1706.05255 \mathrm{v} 3$.

Schulkes, R. M. S. M., Hosking, R. J. \& Sneyd, A. D. 1987 Waves due to a steadily moving source on a floating ice plate. Part 2. J. Fluid Mech. 180, 297-318.

SMORODIN, A. I. 1972 Waves at the fluid surface during the motion of a submerged ellipsoid of revolution. J. Appl. Math. Mech. 36 (1), 137-141. 
Spriggs, J. H., Messiter, A. F. \& Anderson, W. J. 1969 Membrane flutter paradox - an explanation by singular-perturbation methods. AIAA J. 7 (9), 1704-1709.

SYGULSKI, R. 2007 Stability of membrane in low subsonic flow. Intl J. Non-Linear Mech. 42 (1), 196-202.

Tiomkin, S. \& RAVEH, D. E. 2017 On the stability of two-dimensional membrane wings. J. Fluids Struct. 71, 143-163.

Triantafyllou, G. S. 1992 Physical condition for absolute instability in inviscid hydroelastic coupling. Phys. Fluids A 4, 544-552.

Triantafyllou, M. S. \& TRiantafyllou, G. S. 1991 Frequency coalescence and mode localization phenomena: a geometric theory. J. Sound Vib. 150 (3), 485-500.

VEDENEEV, V. V. 2004 Instability of an unbounded elastic plate in a gas flow. Fluid Dyn. 39 (4), 526-533.

VEDENEEV, V. V. 2016 On the application of the asymptotic method of global instability in aeroelasticity problems. Proc. Steklov Inst. Math. 295, 274-301.

Weinfurtner, S., Tedford, E. W., Penrice, M. C. J., Unruh, W. G. \& Lawrence, G. A. 2011 Measurement of stimulated Hawking emission in an analogue system. Phys. Rev. Lett. 106, 021302.

Whitham, G. B. 1999 Linear and Nonlinear Waves. John Wiley \& Sons.

Zhang, R., Qin, H., Davidson, R. C., LiU, J. \& XiaO, J. 2016 On the structure of the two-stream instability-complex G-Hamiltonian structure and Krein collisions between positive- and negative-action modes. Phys. Plasmas 23, 072111. 